\title{
Collimated molecular jets from high-mass young stars: IRAS 18151-1208*
}

\author{
C. J. Davis ${ }^{1}$, W. P. Varricatt ${ }^{1}$, S. P. Todd ${ }^{2}$, and S. K. Ramsay Howat ${ }^{2}$ \\ 1 Joint Astronomy Centre, 660 North A’ohōkū Place, University Park, Hilo, Hawaii 96720, USA \\ e-mail: c.davis@jach.hawaii.edu \\ 2 UK Astronomy Technology Centre, Royal Observatory, Blackford Hill, Edinburgh, EH9 3HJ, UK
}

Received 15 May 2004 / Accepted 22 June 2004

\begin{abstract}
Recent near-IR images of massive star forming regions have revealed two collimated jets in the IRAS 18151-1208 region, one of which is almost a parsec in length (Varricatt et al.). Follow-up high-spectral-resolution echelle spectroscopy and 2-dimensional "integral field" spectroscopy of the associated molecular shock features are presented here. From these data kinematic information and excitation maps are extracted, which show that the two jets are morphologically, kinematically and energetically similar to their counterparts from low mass protostars. The close association between the $\mathrm{H}_{2}$ emission features and the high-velocity $\mathrm{CO}$ emission presented by Beuther et al. also suggests that the $\mathrm{CO}$ represents gas entrained by these two very collimated jets. From the mass and momentum of the molecular gas, and the luminosity of the $\mathrm{H}_{2}$ features, it is clear that the flows must be powered by massive sources. To all intents and purposes, the molecular jets appear to be scaled-up versions of low-mass YSO jets. Collectively, the observations add further support to the idea that massive stars are formed through vigorous disk accretion, and that, while in their earliest stages of evolution, massive protostars drive collimated jets.
\end{abstract}

Key words. molecular data - shock waves - stars: formation - infrared: ISM - infrared: stars - ISM: Herbig-Haro objects

\section{Introduction}

In recent years there has been considerable debate as to whether massive stars form through accretion, like their low-mass counterparts, or through the coalescence of lower-mass stars (see for example the review of Churchwell 2002). Massive young stellar objects (YSOs) are usually found in crowded clusters of many dozens of stars, yet they are also found to be associated with molecular outflows (e.g. Beuther et al. 2002a). Bipolar outflows, and particularly collimated jets, have been used as signposts of disk accretion, and more generally as an indicator that massive young stars form in a way similar to their low mass counterparts (Kumar et al. 2002; Brooks et al. 2003). However, examples of very collimated jets, akin to those seen from low mass young stars, are still rare. Observations are hampered by the considerable distances to massive star forming regions, the increased extinction typically associated with the central objects, and the shorter evolutionary time scales. High-resolution, near-infrared (near-IR) observations offer a potentially power technique for searching for collimated flows (Ayala et al. 1998; Davis et al. 1998; Poras et al. 2000; Shepherd et al. 2000), since extinction is less of a problem than at optical wavelengths, and the high spatial resolution needed to disentangle sources at kilo-parsec distances is attainable.

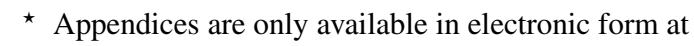
http://www . edpsciences.org
The above arguments prompted us to conduct a shallow, though extensive, near-IR imaging survey of massive star forming regions that showed signs of outflow activity (e.g. highvelocity $\mathrm{CO}$ emission, $\mathrm{H}_{2} \mathrm{O}$ and $\mathrm{CH}_{3} \mathrm{OH}$ masers, etc.). In this survey a number of molecular flows were discovered (Varricatt et al. 2004), flows that may be driven by massive YSOs and may be the massive counterparts of the bipolar outflows driven by low mass YSOs and T Tauri stars. The outflows are traced in molecular hydrogen emission, and in some cases, also in $\mathrm{Br} \gamma$ emission.

From the survey, one rather spectacular multiple-jet system was discovered, which we focus on in this paper. The source region, IRAS 18151-1208, contains two well-defined, collimated outflows. IRAS 18151-1208 is associated with high-velocity $\mathrm{CO}$ emission (Beuther et al. 2002a) and $\mathrm{CH}_{3} \mathrm{OH}$ and $\mathrm{H}_{2} \mathrm{O}$ maser emission (Beuther et al. 2002b). IRAS 18151-1208 itself is embedded in a high-density core (Bronfman et al. 1996), it is bright at far-infrared (FIR) wavelengths and has the FIR colours typical of Ultra-Compact HII (UCHII) regions (Wood $\&$ Churchwell 1989). However, the source was not detected at centimeter-wavelengths $\left(F_{3.6 \mathrm{~cm}}<1 \mathrm{mJy}\right)$ by Sridharan et al. (2002), which indicates the lack of a "substantial" HII region. Instead, the source may be in a pre-UCHII phase; its doublepeaked SED is typical of low-mass Class I YSOs, where the cold dust component, peaking at $\sim 100 \mu \mathrm{m}$, dominates over the warmer dust component at $\sim 20 \mu \mathrm{m}$. The bolometric luminosity of the source, derived from IRAS HiRes photometry, 
Table 1. Observing parameters.

\begin{tabular}{|c|c|c|c|c|}
\hline Source & $R^{a}$ & $\begin{array}{c}\Delta \lambda^{b} \\
\text { (microns) }\end{array}$ & $\begin{array}{l}\mathrm{PA}^{c} \\
(\mathrm{deg})\end{array}$ & $\begin{array}{l}\text { Exp. time }{ }^{d} \\
\quad(\mathrm{~s})\end{array}$ \\
\hline \multicolumn{5}{|l|}{ Echelle spectroscopy: } \\
\hline$\overline{\text { Slit } 1 \text { (NW-SE jet) }}$ & $\sim 14,300$ & $2.115-2.128$ & 134.0 & 500 \\
\hline Slit 2 (NE-SW jet) & $\sim 14,300$ & $2.115-2.128$ & 33.5 & 500 \\
\hline \multicolumn{5}{|l|}{ IFU spectroscopy: } \\
\hline$\overline{\mathrm{IRS} 1}$ & $\sim 1000$ & $1.41-2.50$ & 134.0 & 2880 \\
\hline knots B1-B3 & $\sim 1000$ & $1.41-2.50$ & 134.0 & 1440 \\
\hline knots $\mathrm{C} 1-\mathrm{C} 2$ & $\sim 1000$ & $1.41-2.50$ & 33.5 & 1440 \\
\hline knot X1 & $\sim 1000$ & $1.41-2.50$ & 33.5 & 1440 \\
\hline knot Z1 & $\sim 1000$ & $1.41-2.50$ & 33.5 & 960 \\
\hline \multicolumn{5}{|c|}{ a Spectral resolution. } \\
\hline \multicolumn{5}{|c|}{$b$ Wavelength coverage of grism/grating. } \\
\hline \multicolumn{5}{|c|}{$\begin{array}{l}c \text { Position angle of echelle long-slit or IFU long }\left(6.0^{\prime \prime}\right) \text { axis, mea- } \\
\text { sured E of N. }\end{array}$} \\
\hline \multicolumn{5}{|c|}{$d$ Total exposure time spent on-source. } \\
\hline
\end{tabular}

is $\sim 20000 L_{\odot}$ (Sridharan et al. 2002). The kinematic distance to IRAS 18151-1208, based on CS observations, is $3.0 \mathrm{kpc}$ (Brand \& Blitz 1993).

Here we present images of the $\mathrm{H}_{2}$ jets, together with high-spectral-resolution echelle and intermediate-spectralresolution Integral Field spectroscopy. These data yield detailed kinematic and excitation properties which we compare with those of jets and molecular outflows from low-mass YSOs, as well as with models of bipolar outflows. Our general aim is to establish whether these jets are simply scaled-up versions of the low-mass YSO outflows, or whether they are quantitatively different.

\section{Observations}

As part of an extensive near-IR imaging survey of high-mass YSO candidates (Varricatt et al. 2004), narrow-band $\mathrm{H}_{2}(\lambda=$ $2.122 \mu \mathrm{m}, \Delta \lambda(F W H M)=0.020 \mu \mathrm{m})$ and broad-band $\mathrm{K}(\lambda=$ $2.20 \mu \mathrm{m}, \Delta \lambda(F W H M)=0.34 \mu \mathrm{m})$ images of IRAS 181511208 were obtained on 28 May 2003 (UT) at the U.K. Infrared Telescope (UKIRT) using the facility near-IR imager UFTI (Roche et al. 2002). UFTI is equipped with a Rockwell Hawaii $1024 \times 1024 \mathrm{HgCdTe}$ array and has a plate scale of $0.091^{\prime \prime}$ per pixel, resulting in a field of view of 92 ". Nine-point "jittered" mosaics were obtained in each filter. Standard reduction techniques were employed. These included null debiassing, bad-pixel masking, dark subtraction, flat-field creation (from the jittered source frames themselves) and division, feature detection and matching between object frames, and resampling.

Echelle spectra in $\mathrm{H}_{2} \quad 1-0 \mathrm{~S}(1)$ emission $\left(\lambda_{\text {vac }}=\right.$ $2.1218334 \mu \mathrm{m}$; Bragg et al. 1982) were obtained on 29 May 2003 using the cooled grating spectrometer CGS 4 (Mountain et al. 1990). The instrument is equipped with a $256 \times 256$ pixel InSb array; the pixel scale is $0.41^{\prime \prime} \times 0.88^{\prime \prime}$ (0.41" in the dispersion direction). A 2-pixel-wide slit was used, resulting in a velocity resolution of $\sim 21( \pm 2) \mathrm{km} \mathrm{s}^{-1}$

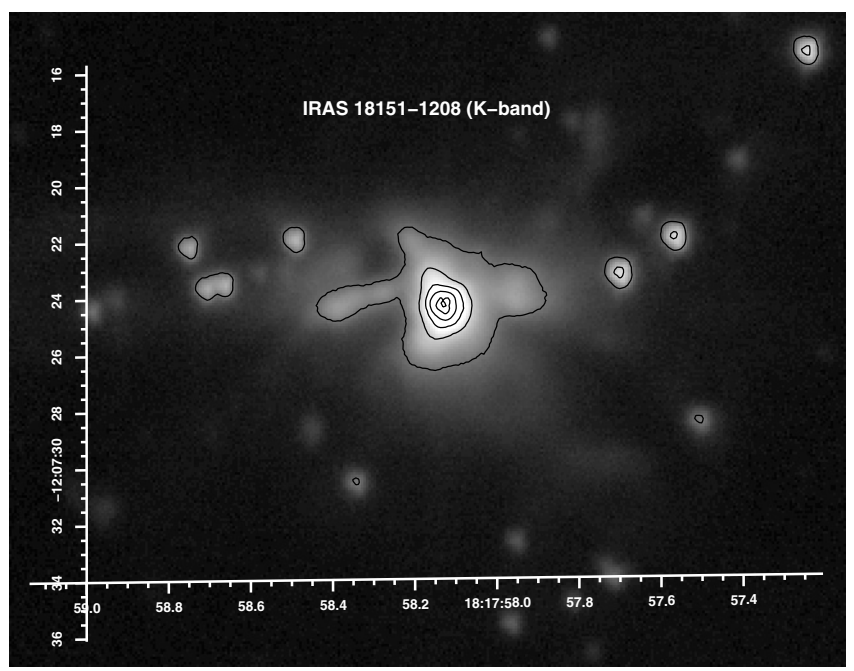

Fig. 1. $K$-band image of the source region, showing the single, bright near-IR peak associated with IRAS 18151-1208 which we denote IRS 1 . The coordinates of IRS 1 are RA(2000): $18^{\mathrm{h}} 17^{\mathrm{m}} 58^{\mathrm{s}} .1$, $\operatorname{Dec}(2000):-12^{\circ} 07^{\prime} 25^{\prime \prime}$. Contours are arbitrary.

(measured from Gaussian fits to sky lines). Data at two locations were obtained (Table 1), with the slit orientated along the northwest-southeast outflow denoted by knots B-C in Fig. 2 (slit 1) and through the northeast-southwest flow labeled X-Y$\mathrm{Z}$ in Fig. 2 (slit 2). Slit 1 was centred ("peaked-up") on the $K$-band continuum peak (Fig. 1) assumed to be associated with the IRAS peak and outflow source that powers the northwestsoutheast jet and knots A, B and C.

At each position a "sky" exposure was followed by five onsource "object" exposures, the former being subtracted from all object frames. Each spectral image was bias subtracted and flat-fielded. Sky-subtracted object frames were then co-added into reduced "groups" (one group frame per slit position). Each reduced group spectral image was subsequently wavelength calibrated using OH sky lines (Oliva \& Origlia 1992; Davis et al. 2001a); the first "raw object frame" observed at each slit location (the first frame in each group) was used as a reference frame in each case. The Starlink software packages Figaro and Kappa, used to do this, also correct for distortion along the columns in each image (i.e. along arc or sky lines), via a polynomial fit to the sky lines in each row. The relative velocity calibration along the full length of the slit, measured from Gaussian fits to sky lines in "velocity-calibrated and distortion-corrected" raw frames, is estimated to be accurate to $\leq 6 \mathrm{~km} \mathrm{~s}^{-1}$ (all velocities are with respect to the Local Standard of Rest [LSR]). Instrumental flexure over the duration of the observation is unlikely to affect this calibration (e.g. Davis et al. 2001a). A spectrum of an A0V bright standard star (HIP87813) was obtained and reduced in a similar fashion; the extracted spectrum was "grown" into a 2D image so that division by this image would correct the reduced IRAS 18151-1208 spectral images for telluric absorption.

Finally, Integral Field Unit (IFU) spectra at five positions in IRAS 18151-1208 were secured, using the UKIRT Imager-Spectrometer, UIST (Ramsay Howat et al. 2000), on 30 May, 4 June, 4 August and 22 August 2003 (UT). The IFU 


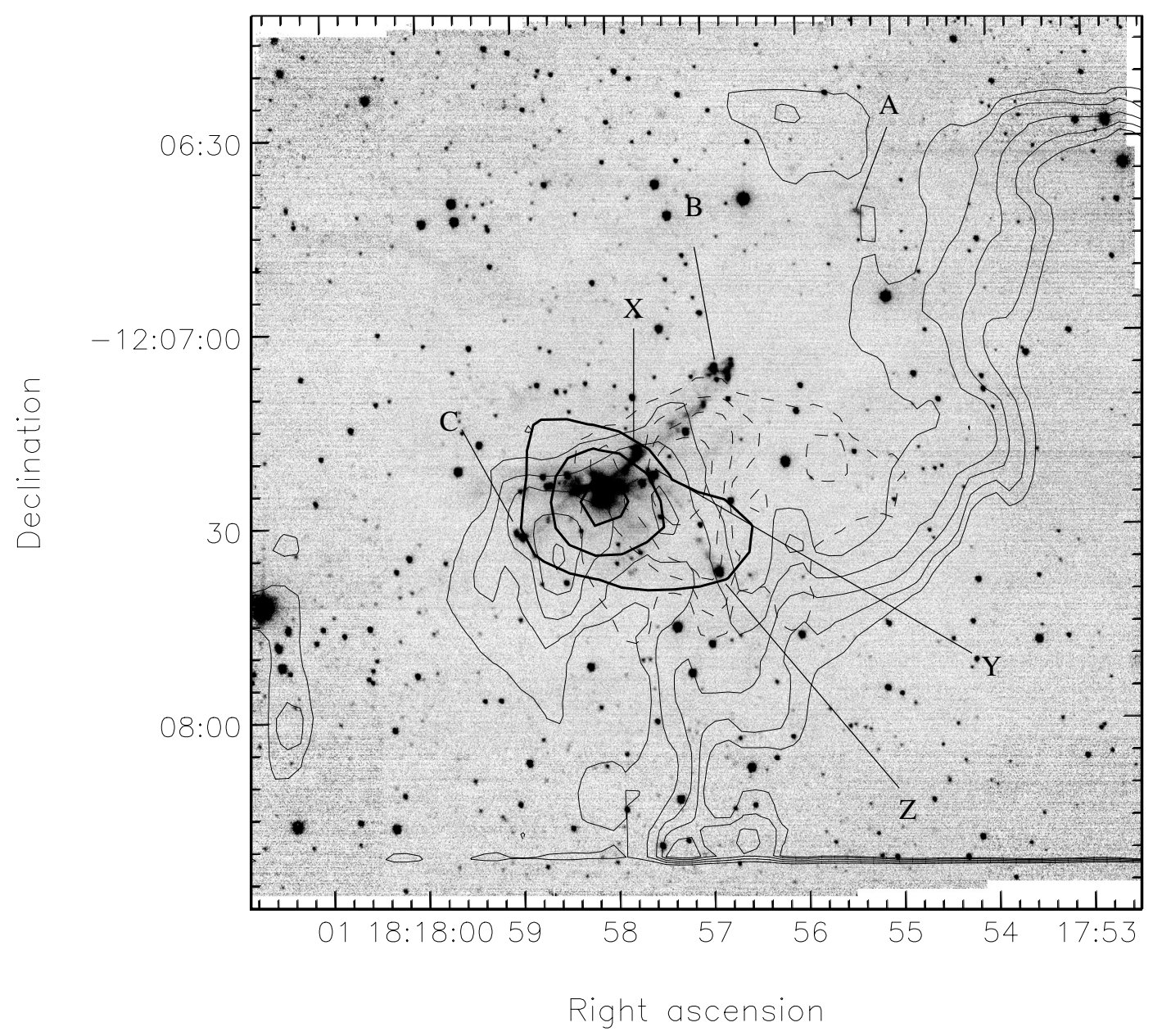

Fig. 2. An UFTI $2.12 \mu \mathrm{m}$ narrow-band image of IRAS 18151-1208 with CO $J=2-1$ line and $1.2 \mathrm{~mm}$ continuum emission superimposed as contours (from Beuther et al. 2002a,b): $1.2 \mathrm{~mm}$ data are plotted with thick, full contours; blue-shifted CO with thin, full contours and red-shifted $\mathrm{CO}$ with thin, dashed contours.

provides moderate-resolution spectroscopy over a $3.3^{\prime \prime} \times 6.0^{\prime \prime}$ rotatable field, with a pixel scale of $0.24^{\prime \prime} \times 0.12^{\prime \prime}$. The image slicing mirror comprises 18 segments that, on the sky, represent horizontal adjacent "slitlets", each 0.24 " $\times 6.0$ " in size. 14 adjacent slitlets are usable, giving the field-of-view noted above. The IFU can be used with any of the installed grisms; an intermediate-resolution HK grism was used in this case. Observing parameters are listed in Table 1.

The IFU data were reduced using pipeline software developed as part of the ORAC project (Todd et al. 2003), although some post-pipeline reduction (division by a standard star, flux calibration and extraction of images and spectra) was carried out with generic Figaro routines. Briefly, a bad pixel mask was applied to the raw data. Data were flat-fielded using an exposure of an internal black-body lamp, and sky subtracted with exposures on nearby blank sky regions. The 14 "mini" spectral images projected onto the array by the IFU mirror segments (one mini-spectral-image for each slitlet) were individually wavelength-calibrated using an argon arc spectrum, a template line-list and an automated arcline-fitting routine. In the resulting "scrunched" spectral images the 14 2D spectra appear in adjacent panels. The 14 panels are subsequently extracted and combined into a 3D datacube that has dimensions of 14 pixels in $\mathrm{X}\left(0.24^{\prime \prime}\right.$-wide slitlets $\times 14$ slitlets corresponds to $3.3^{\prime \prime}$ ), approximately 50 pixels in $\mathrm{Y}$ (the length of each slitlet on the sky; 50 pixels corresponds to $\sim 6^{\prime \prime}$ ) and 1024 pixels in " $\mathrm{T}$ ", the third, dispersion (wavelength) axis. Thus, by extracting data over a narrow wavelength range in the T-direction, images in any spectral line or over any wavelength range (between 1.4 and $2.45 \mu \mathrm{m}$ ) can be obtained. Similarly, by extracting and co-adding spectra over spatial regions in $\mathrm{X}$ and $\mathrm{Y}$, a spectrum covering a specified area within the full $3.3^{\prime \prime} \times 6.0^{\prime \prime}$ field-of-view of the IFU can be obtained.

Data for the emission-line features in IRAS 18151-1208 and for the A0V standard HIP 87813 were obtained in the same manner (although the exposure times were $240 \mathrm{~s}$ and $7 \mathrm{~s}$ respectively) and initially reduced in exactly the same way, up to the point where the cube is created. For the standard, however, five or seven (depending on the seeing) spectra from the scrunched spectral image were extracted and coadded; photospheric absorption lines, notably $\mathrm{Br} \gamma$, were removed from the resulting spectrum before it was expanded into a data cube. The cube, corrected for the black-body function of the standard's spectral type, was then divided into each of the IRAS 18151-1208 data cubes to correct for telluric absorption and to flux-calibrate the data. Note, however, that much of the data were taken 
Table 2. $\mathrm{H}_{2}$ line ratios, the excitation temperature, ortho-para ratio and the estimated extinction for each knot/sub-region observed with the IFU.

\begin{tabular}{ccccccc}
\hline \hline Knot & $\begin{array}{c}\mathrm{Area}^{a} \\
(\operatorname{arcsec})\end{array}$ & $\begin{array}{c}A_{\mathrm{v}}^{b} \\
(\mathrm{mag})\end{array}$ & $\begin{array}{c}T_{\mathrm{ex}}^{c} \\
(\mathrm{~K})\end{array}$ & $\frac{1-0 \mathrm{~S}(1)}{2-1 \mathrm{~S}(1)}^{d}$ & $\frac{1-0 \mathrm{~S}(1)}{3-2 \mathrm{~S}(3)}^{d}$ & $\phi_{1}{ }^{e}$ \\
\hline $\mathrm{B} 1$ & $0.72 \times 0.48$ & 28 & $2529\left(\frac{ \pm 230}{-223}\right)$ & $8.1( \pm 1)$ & - & $2.9( \pm 0.9)$ \\
$\mathrm{B} 2$ & $0.72 \times 0.84$ & 30 & $2465\left(\frac{ \pm 115}{-115}\right)$ & $7.0( \pm 1)$ & $19.4( \pm 7)$ & $2.8( \pm 0.3)$ \\
$\mathrm{B} 3$ & $0.96 \times 0.60$ & 20 & $2605\left(\frac{ \pm 144}{-140}\right)$ & $9.5( \pm 2)$ & $38.6( \pm 13)$ & $3.1( \pm 0.3)$ \\
$\mathrm{B} 4$ & $0.48 \times 0.36$ & 16 & $2004\left(\frac{+123}{-125}\right)$ & $9.7( \pm 6)$ & $>31$ & $3.0( \pm 0.8)$ \\
$\mathrm{B} 7$ & $1.20 \times 0.72$ & 19 & $2286\left(\frac{+73}{-74}\right)$ & $9.4( \pm 1)$ & $52.5( \pm 46)$ & $3.0( \pm 0.2)$ \\
$\mathrm{C} 1$ & $1.20 \times 1.08$ & 23 & $2294\left(\frac{+107}{-108}\right)$ & $10.1( \pm 2)$ & $13.5( \pm 7)$ & $2.7( \pm 0.2)$ \\
X1a & $0.72 \times 0.72$ & 17 & $2298\left(\frac{+34}{-43}\right)$ & $9.8( \pm 1)$ & $45.3( \pm 23)$ & $2.8( \pm 0.1)$ \\
X1b & $0.96 \times 0.60$ & 20 & $2407\left(\frac{+32}{-39}\right)$ & $9.4( \pm 1)$ & $42.8( \pm 19)$ & $2.8( \pm 0.1)$ \\
Z1a & $1.44 \times 0.72$ & 10 & $2031\left(\frac{+46}{-43}\right)$ & $10.0( \pm 1)$ & - & $2.9( \pm 0.1)$ \\
Z1b & $1.44 \times 0.72$ & 18 & $2038\left(\frac{+57}{-57}\right)$ & $11.2( \pm 2)$ & $31.8( \pm 16)$ & $2.6( \pm 0.1)$ \\
\hline
\end{tabular}

${ }^{a}$ Area in each IFU frame over which spectra were extracted and summed (in arcseconds). Each region is labeled in Fig. 8. Knots B1-B7 and C1 are reasonably discrete features, while X1a and $\mathrm{X} 1 \mathrm{~b}$ represent the head and flanks of one bow shock, and Z1a and $\mathrm{Z} 1 \mathrm{~b}$ the head and flanks of another.

${ }^{b}$ Visual extinction derived from linear least-squares fits to excitation diagrams plotted from the line intensities given in Appendix A (see e.g. Fig. 9).

${ }^{c} \mathrm{H}_{2}$ excitation temperature derived from maximum-likelihood method fits to the line intensities given in Appendix A (errors are given in brackets).

${ }^{d}$ Line intensity ratios derived from the $\mathrm{H}_{2} 1-0 \mathrm{~S}(1), 2-1 \mathrm{~S}(1)$ and 3-2 S(3) lines (at $2.121 \mu \mathrm{m}, 2.247 \mu \mathrm{m}$ and $2.201 \mu \mathrm{m}$ ). Values are corrected for extinction assuming a law of the form $\log \left(I / I_{\mathrm{o}}\right)=$ $-0.4 A_{\mathrm{v}}\left(\lambda / \lambda_{\mathrm{v}}\right)^{-1.74}$, where $A_{\mathrm{v}}$ and $\lambda_{\mathrm{v}}$ are the extinction and wavelength at $0.6 \mu \mathrm{m}$.

e The $\mathrm{H}_{2}$ ortho-para ratio for the $v=1$ states. $\phi_{1}$ is derived from the 1-0 S(0), 1-0 S(1) and 1-0 S(2) integrated line intensities (at $2.223 \mu \mathrm{m}, 2.121 \mu \mathrm{m}$ and $2.033 \mu \mathrm{m}$ ) following the method of Smith et al. (1997).

through thin cirrus, so the absolute flux calibration is only accurate to within a factor of $\sim 2$. Specific line intensities are best derived from the line ratios listed in Table 2, combined with $\mathrm{H}_{2}$ 1-0 S(1) surface brightnesses taken from Fig. 3 below.

\section{Results and analysis}

\section{1. $\mathrm{H}_{2}$ imaging}

Images of the IRAS 18151-1208 region are presented in Figs. 1-3. A bright central peak, denoted IRS 1, is evident in the $K$-band image in Fig. 1 . The position of this IR peak coincides, to within $1^{\prime \prime}-2^{\prime \prime}$, with a $6.7 \mathrm{GHz} \mathrm{CH}_{3} \mathrm{OH}$ maser and the strongest dust continuum peak in the region, seen at $450 \mu \mathrm{m}, 850 \mu \mathrm{m}$ and $1.2 \mathrm{~mm}$ (Williams et al. 2004; Beuther et al. 2002b). The maser, which peaks at low radial velocities, is probably associated with the IRS 1 stellar envelope or disk. The $1.2 \mathrm{~mm}$ data of Beuther et al. are reproduced in Fig. 2. The IRAS point-source catalogue position of IRAS 18151-1208 is offset by $-15^{\prime \prime},+3$ " from IRS1, although the error ellipse is $25^{\prime \prime} \times 7^{\prime \prime}$ with a position angle of $87^{\circ}$, so it is likely that the bulk of the IRAS flux also derives from IRS 1. MSX resolved one source in the region (among considerable filamentary nebulosity) situated at RA(2000): $18^{\mathrm{h}} 17^{\mathrm{m}} 58^{\mathrm{s}} .0$, Dec(2000): $-12^{\circ} 07^{\prime} 29^{\prime \prime}$, coincident with IRS1 to within $\sim 5^{\prime \prime}$.

Two distinct outflows are evident in the $\mathrm{H}_{2}$ images in Figs. 2 and 3. In Fig. 2 we compare our $\mathrm{H}_{2}$ observations with the $\mathrm{CO}$ outflow maps of Beuther et al. (2002a); in Fig. 3, the $\mathrm{H}_{2}$ data are shown in more detail. The features labeled A, B and C are presumably associated with the same collimated flow, even though they are not on precisely the same axis. The slight misalignment of some of the knots probably results from emission being excited along the edges of a tubular cavity, or from the flow direction varying slightly with time. Given the general alignment of these features with the bright IR nebulosity (the emission peak is marked with a black square in Fig. 3) it seems likely that IRS1 or an adjacent source in the central cluster powers the outflow that excites the $\mathrm{H}_{2}$ features. Knot A probably represents an initial outflow phase, with knots B1-B4 being separate "mini-bow shocks" associated with a second, later burst of outflow. The clumpy morphology of B1-B4 is commonly observed in $\mathrm{H}_{2}$ images of bow shocks in jets from lowmass YSOs, where the structure is thought to result from either instabilities in the overall shock structure, or from directional variability in the jet itself (e.g. Blondin et al. 1989; Raga 1993; Volker et al. 1999; Downes \& Ray 1999). Knots B5-B8 trace the jet axis back toward the IRS 1 source, while knots $\mathrm{C} 1-\mathrm{C} 5$ mark the counter-flow. At a distance of $3 \mathrm{kpc}$ to IRAS 181511208 , the length of the jet, from the source to knot A, is at least $0.85 \mathrm{pc}$ (note that we did not find a counter-part of knot A to the southeast of IRS1).

The second jet evident in Fig. 3 is delineated by knots $\mathrm{X}$, $\mathrm{Y}$ and $\mathrm{Z}$. The powering source of this flow is not obvious, although judging from the orientation of the "bow shaped features" Z1, Y1 and possibly X1, it probably lies between knots $\mathrm{Z} 1$ and $\mathrm{Y} 1$. There is no bright $K$-band source in this region, although the (sub)millimeter dust-continuum peak that coincides with IRS 1 in Fig. 2 does extend roughly $20^{\prime \prime}-30^{\prime \prime}$ to the southwest (Beuther et al. 2002b; Williams et al. 2004), suggesting the presence of a second, weaker, unresolved source between knots Z1 and Y1.

Beuther et al. (2002a) have mapped high-velocity CO emission in IRAS 18151-1208. Their maps indicate the presence of at least one bipolar molecular outflow in the region. Comparison of their data with our $\mathrm{H}_{2}$ image in Fig. 2 shows that IRS 1 is precisely situated between the southeastern blueshifted lobe and the northwestern red-shifted lobe of the $\mathrm{CO}$ flow. This bipolar flow is presumably associated with the A-B-C jet. However, note also the second blue-shifted CO peak $\sim 15^{\prime \prime}$ to the southwest of IRS 1 . This peak coincides with, and is elongated in the same direction as, the second $\mathrm{H}_{2}$ jet seen here (knots X-Y-Z). So although higher-resolution $\mathrm{CO}$ data would obviously help unravel the $\mathrm{CO}$ flow components in IRAS 18151-1208, the $\mathrm{H}_{2}$ emission features in both jets do seem to be associated with collimated CO flow lobes, as is typically the case in low-mass outflows (e.g. Davis \& Eislöffel 1995; Lee et al. 2000). 


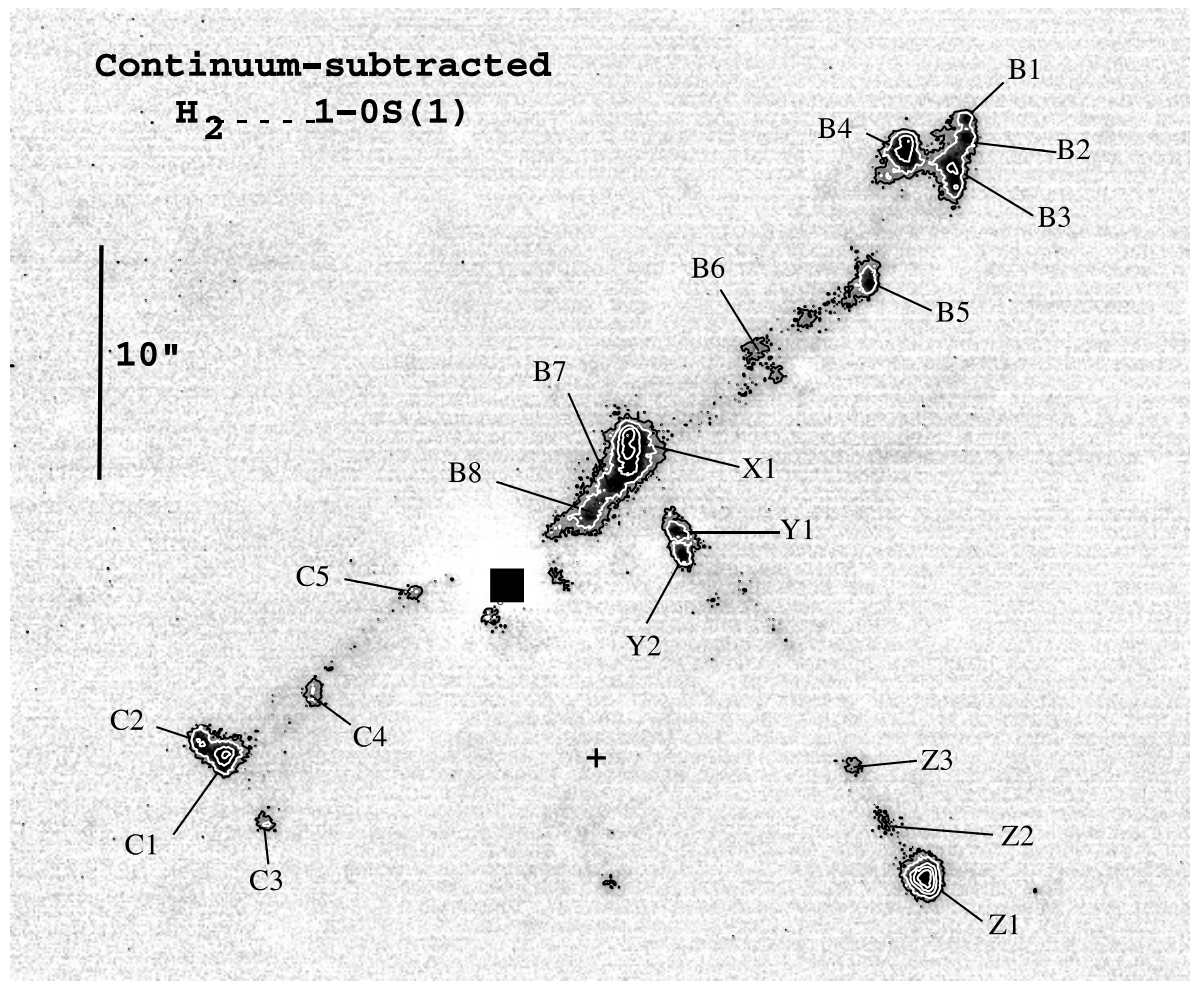

Fig. 3. A contour plot showing the central region of the $\mathrm{H}_{2}$ image in more detail. The image has been continuum-subtracted to show only the line-emission features. The contours measure $2.5,5,12,20$ and $30 \times 10^{-18} \mathrm{~W} \mathrm{~m}^{-2} \operatorname{arcsec}^{-2}$. The black square marks the location of the $K$-band continuum peak, IRS 1.

\subsection{Echelle spectroscopy of $\mathrm{H}_{2}$ 1-O S(1) emission}

Echelle spectra were obtained along the axes of the two jets noted above. For jet A-B-C the slit (slit 1) was centred on the continuum emission peak IRS 1; slit 2 was aligned through knots X1, Y1 and Z1. Position-Velocity (P-V) diagrams, showing the line-of-sight radial velocity at each position along the slit, are shown in Fig. 4.

From features B2, B6, B7 and B8 (slit 1) the overall emission is red-shifted, as one would expect for knots associated with the red lobe of the $\mathrm{CO}$ outflow (Beuther et al. 2002a). The double-peaked profiles observed toward B2 could be due to the bow-shaped morphology of the shock front (e.g. Hartigan et al. 1987; Völker et al. 1999; Davis et al. 2001a). The $\mathrm{H}_{2}$ profile from B2 peaks at LSR velocities of $38.0 \mathrm{~km} \mathrm{~s}^{-1}$ and $73.7 \mathrm{~km} \mathrm{~s}^{-1}$ (red-shifted by $6.0 \mathrm{~km} \mathrm{~s}^{-1}$ and $41.7 \mathrm{~km} \mathrm{~s}^{-1}$ with respect to the systemic velocity) and the overall profile width at the $10 \%$ peak-intensity level is $\sim 88 \mathrm{~km} \mathrm{~s}^{-1}$.

In a "magnetically-mediated" C-type shock, $\mathrm{H}_{2}$ is dissociated at shock speeds in excess of $\sim 50 \mathrm{~km} \mathrm{~s}^{-1}$ : the limit to the full line width (at zero intensity) in a C-type bow shock is essentially twice this speed, or less than this if the flow is inclined out of the plane of the sky (Smith et al. 2003). The spatial extent of the $\mathrm{H}_{2}$ jet (on the sky) and the modest overlap of the CO outflow lobes (Beuther et al. 2002a) suggest an inclination angle close to the plane of the sky. Thus, $\mathrm{H}_{2}$ line widths of $80-100 \mathrm{~km} \mathrm{~s}^{-1}$ are possible. Indeed, broad line widths are $e x$ pected from a high-velocity bow if the bow shock geometry is the dominant mechanism that shapes the line profile, since the most intense $\mathrm{H}_{2}$ emission will be excited as close to the bow shock cap as possible, where post-shock velocities are at their highest and the deflection of the post-shock gas away from the symmetry axis is at its greatest. Only dissociation in the bow cap itself limits the width of the observed line profile. The $m e$ dian velocity, in this case $\sim 20 \mathrm{~km} \mathrm{~s}^{-1}$ with respect to the systemic, is then a function of the inclination angle of the flow to the line of sight, as well as the velocity of the pre-shock gas (higher pre-shock gas velocities will shift the whole line profile to higher red-shifted velocities).

Some (or all) of the high-velocity $\mathrm{H}_{2}$ emission in $\mathrm{B} 2$ could be associated with the jet that drives the bow shock, rather than the bow itself. This interpretation was recently used to explain similar echelle observations of the prototypical bow shock $\mathrm{HH} 7$ (Smith et al. 2003). In HH7, double-peaked $\mathrm{H}_{2}$ profiles peaking at $\sim 10 \mathrm{~km} \mathrm{~s}^{-1}$ and $90 \mathrm{~km} \mathrm{~s}^{-1}$ were observed. The lowvelocity component (as well as the overall bow morphology and gas excitation) were convincingly modeled with a C-type bow shock. But the high-velocity component was assumed to derive from the jet itself, specifically from the Mach disk at the interface between the jet and the swept-up gas behind the bow shock. This interpretation was supported by the fact that the high-velocity peak is much weaker than the low-velocity peak in HH 7. It is also spatially less extended, and is displaced behind (upwind of) the high-velocity gas. In the IRAS 181511208 bow, however, the low and high-velocity components are similar in extent and intensity, as one would expect for emission from the two sides of a geometrically uniform cone or parabola. Also, the high-velocity emission in $\mathrm{HH} 7$ is situated very close to the bow shock cap: in IRAS 18151-1208 the 

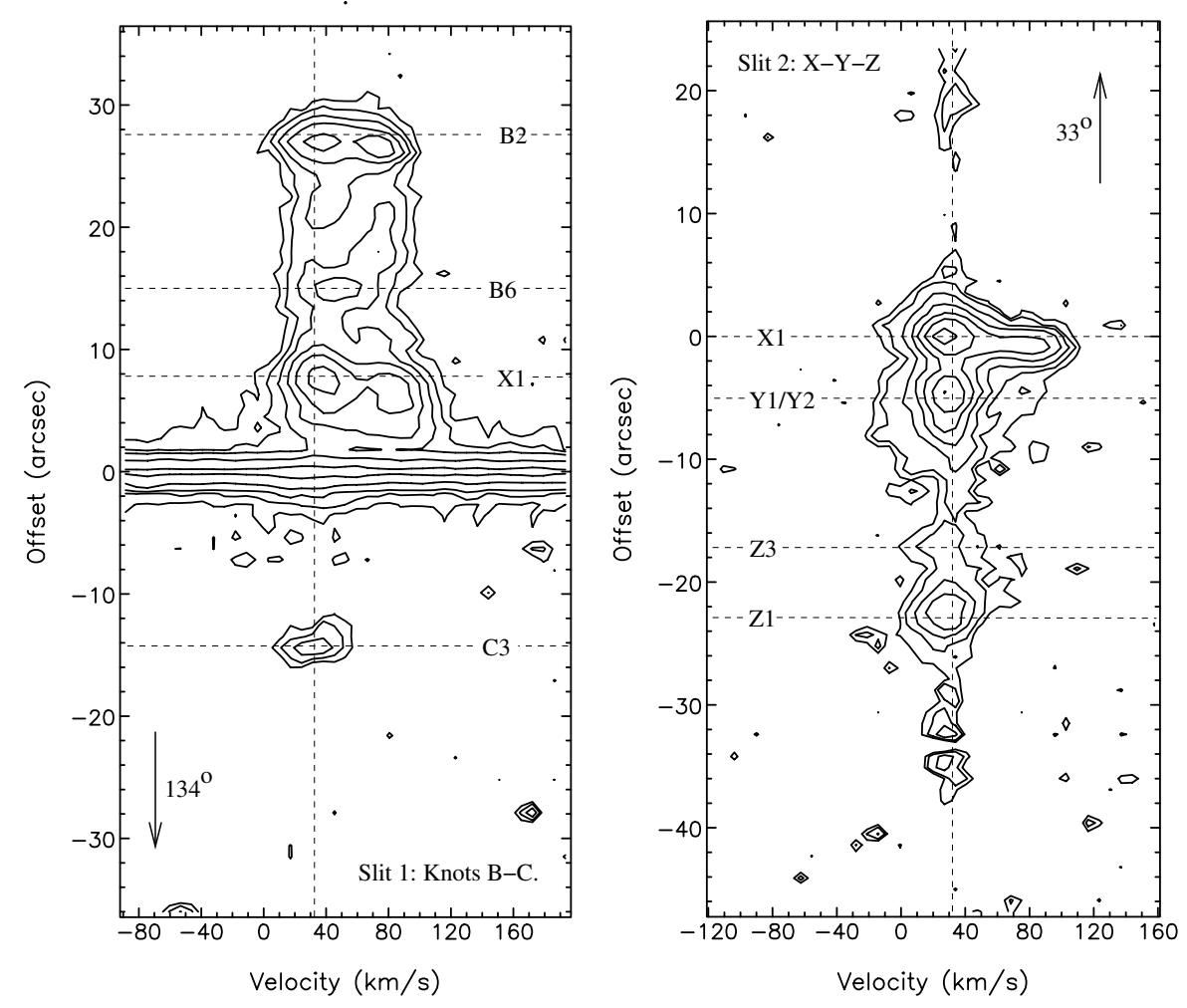

Fig. 4. Echelle spectral images in $\mathrm{H}_{2}$ 1-0 S(1) emission. Slit 1 runs along the northwest-southeast flow (through IRS1 and knots B2-B8 and $\mathrm{C} 3$ ), while slit 2 runs through the bright knots in the orthogonal flow denoted by knots $\mathrm{X}, \mathrm{Y}$ and $\mathrm{Z}$. The positions of brighter knots along each slit are labeled; the vertical dashed line marks the systemic LSR velocity of $32 \mathrm{~km} \mathrm{~s}^{-1}$. Contours for slit 1 start at $5 \sigma$ and increase in multiples of 2; contours for slit 2 start at $2.5 \sigma$ and increase in multiples of 2.

spatial offset between the two velocity components is $\sim 4^{\prime \prime}$, or about $12000 \mathrm{AU}$. This upwind (southeast) offset of the highvelocity component is expected if the emission is excited in the far-side wings of the bow which, given the inclination of the red-shifted outflow lobe away from the observer, would then be projected slightly behind the near-side bow wings.

Thus, a high-velocity bow shock interpretation is favoured for the profiles observed in knot B2. The double-peaked profiles observed closer to IRS 1 (near knot X1), and the similar offset upwind of the high-velocity peak with respect to the lowvelocity peak, can probably be explained in the same way.

Slit 2 passes along the orthogonal jet X-Y-Z (Fig. 4). At all locations along the slit, the $\mathrm{H}_{2}$ emission peaks are at rest with respect to the systemic velocity (the red-shifted emission near $\mathrm{X} 1$ is presumably from the A-B-C jet, since it is not detected elsewhere along slit 2 and red-shifted $\mathrm{H}_{2}$ is detected along much of slit 1). Presumably the X-Y-Z flow also lies very close to the plane of the sky. The profiles are generally narrower than in the A-B-C jet; for $\mathrm{Z} 1$ and $\mathrm{Y} 1 / \mathrm{Y} 2$ the profile widths at the $10 \%$ intensity level are $71 \mathrm{~km} \mathrm{~s}^{-1}$ and $57 \mathrm{~km} \mathrm{~s}^{-1}$ respectively. As noted earlier, in each bow shock the $\mathrm{H}_{2}$ profiles should be as wide as about twice the shock speed limit. The narrower line profiles in the X-Y-Z flow therefore suggest a lower speed limit: in a J-shock (i.e. one without magnetic cushioning), emission will be excited at velocities over the range $+25 \mathrm{~km} \mathrm{~s}^{-1}$ to $-25 \mathrm{~km} \mathrm{~s}^{-1}$, leading to line profile widths near the apex of the bow of $\sim 50 \mathrm{~km} \mathrm{~s}^{-1}$. J-type bow shock physics, caused by a higher ionisation fraction in the pre-shock gas or a weaker magnetic field, may therefore better describe the observed data in slit 2. However, it is worth noting that [FeII] emission, often regarded as a signpost of J-type shocks (e.g. Gredel 1996), is not observed in the IFU data described below for any of the knots observed (B, C, X or Z).

Finally, we investigate whether $\mathrm{H}_{2}$ line emission is traced all the way back to the IRS 1 source continuum position in slit 1. In high-spectral-resolution observations of embedded low-mass YSOs, emission is usually detected coincident (to within $100 \mathrm{AU}$ ) with the continuum peak (Davis et al. 2001b; Takami et al. 2004). In comparison to imaging and spectroscopy at lower spectral resolution, in echelle data the shot noise associated with the continuum emission near the star is reduced considerably, facilitating the detection of weak line emission. The large CGS4 pixels (0.9" along the slit direction) and distance to IRAS 18151-1208 hamper our analysis of the small-scale structure at the base of the flow. Nevertheless, after fitting and subtracting the continuum spectrum from the P-V diagram in Fig. 4 we do see faint line emission within 1" of the source continuum position (Fig. 5).

As with molecular hydrogen emission line (MHEL) regions at the bases of flows from low-mass YSOs, the brightest emission near IRS 1 is excited at the base of the blue-shifted flow lobe. The emission is only moderately blue-shifted, by $\sim 6 \mathrm{~km} \mathrm{~s}^{-1}$ with respect to the systemic velocity, and the line profile is relatively narrow, $F W H M \sim 47 \mathrm{~km} \mathrm{~s}^{-1}$. The $\mathrm{H}_{2}$ also derives from a fairly discrete feature, i.e. one which is unconnected to other bows and knots further downwind. Mildly 


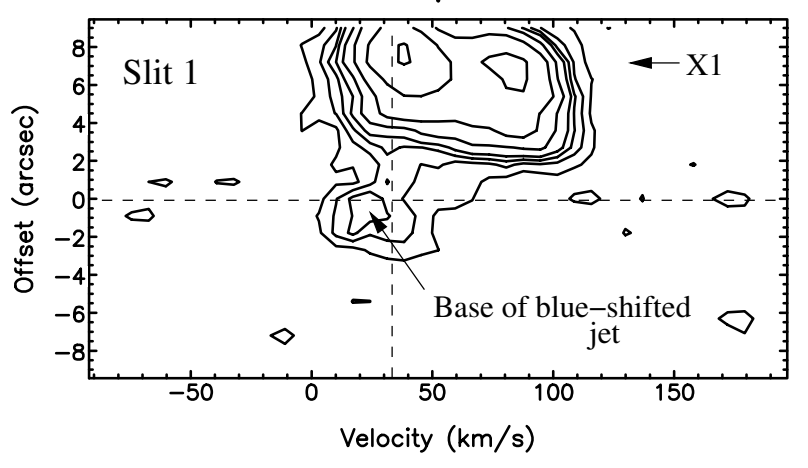

Fig. 5. Echelle spectral image near the IRS 1 source in slit 1 . The continuum emission from IRS 1 has been fitted and removed to show the weak $\mathrm{H}_{2}$ 1-0 S(1) emission at the base of the jet (increasing offsets are to the northwest). The horizontal dashed line marks the continuum position; the vertical dashed line indicates the systemic LSR velocity of $32 \mathrm{~km} \mathrm{~s}^{-1}$. Contours are $2 \sigma, 4 \sigma, 6 \sigma, 8 \sigma, 12 \sigma, 20 \sigma, 40 \sigma$ and $80 \sigma$ (where $1 \sigma$ is the rms noise in the rows where continuum was subtracted).

red-shifted emission is also detected near IRS 1 . The emission extends to the northwest of the source continuum position (positive offsets in Fig. 5), linking the star to the rest of the redshifted flow lobe. The red-shifted MHEL emission is probably weaker than the blue-shifted knot because of increased obscuration from a dense, extended envelope around IRS 1 (this is thought to be the reason why red-shifted $\mathrm{H}_{2}$ is rarely observed toward low-mass YSOs; e.g. Davis et al. 2001b).

Like the MHEL regions in low-mass YSO flows, the molecular emission seen at the base of the IRAS 18151-1208 jet could be excited in ambient gas entrained at the base of a collimated jet. In their [FeII] observations of low-mass YSO jets, Pyo et al. (2002) and Davis et al. (2003) did find a highervelocity, more finely collimated, ionised flow component at the base of each jet observed. The feature in each case is referred to as the Forbidden Emission Line, or FEL, region. Notably, they also found that the ionised FEL flow component typically carries sufficient momentum to drive the MHEL component traced in $\mathrm{H}_{2}$ emission.

Alternatively, the $\mathrm{H}_{2}$ emission could derive from the wind itself. $\mathrm{H}_{2}$ could be excited in the outer layers of a disk wind (e.g. Cabrit et al. 1999; Garcia et al. 2001). Flow streamlines that originate from the outer parts of the disk (disk radii $>1 \mathrm{AU}$ ) may consist of largely, if not entirely, molecular material (Safier 1993). Here, gas temperatures may reach only a few thousand Kelvin; $\mathrm{H}_{2}$ may then survive acceleration from the disk surface. In their disk-wind models, Safier (1993) suggest that the line cooling rate from $\mathrm{H}_{2}$ will sharply rise within the first few AU along the jet axis, then steadily drop off with distance from the source, out to a few hundred or a thousand AU. Such a thermal pattern might produce the offset of the molecular line-emission knots typically seen in MHEL regions associated with low-mass YSOs. It may also explain the offset of the blue peak seen here in Fig. 5. Offsets of a few tens, a few hundred or even a few thousand AU could be possible, depending on the specific conditions in the flow.
$\mathrm{X}$-wind models have yet to treat the conditions in the wideangled component of the flow, although the gas here is expected to be warm and only partially ionised (Shang et al. 2002), ideal conditions, perhaps, for the excitation of $\mathrm{H}_{2}$.

Either way, molecular line cooling does seem to be important in regions at the bases of jets from low mass YSOs; our new observations of IRAS 18151-1208 suggest that this may also be the case for jets from massive YSOs.

\section{3. $\mathrm{H}$ - and $\mathrm{K}$-band Integral field spectroscopy}

\subsubsection{The IRS1 source region}

IFU spectroscopy of the IRS 1 region is shown in Figs. 6 and 7. Figure 6a shows the emission integrated over the same wavelength range as the broad-band $K$ filter used in Fig. 1 . The IFU data clearly show the IRS 1 peak, together with some of the nebulosity evident in Fig. 1.

The remaining three panels in Fig. 6 show continuumsubtracted images in $\mathrm{Br} \gamma, \mathrm{H}_{2} 1-0 \mathrm{~S}(1)$ and [FeII] $1.644 \mu \mathrm{m}$ emission. Continuum-subtraction in these images is nearperfect, since the line and continuum data were acquired at the same time and under the same observing conditions, and because the colour of the continuum is accounted for by averaging continuum data extracted from either side (in wavelength) of the line emission. Thus, the $\mathrm{Br} \gamma$ emission toward the IRS 1 peak is real. The $\mathrm{Br} \gamma$ is probably predominantly associated with accretion, as is often assumed to be the case in spectra of T Tauri stars and low-mass Class I YSOs (e.g. Najita et al. 1996a; Folha \& Emerson 2000; Davis et al. 2001b), although emission from dense, high-excitation regions at the very base of the jet could contribute, as could emission from a compact HII region. In both cases, the emission would not be spatially resolved. The $\mathrm{H}_{2}$ and [FeII] is, by comparison, only observed in the jet, from the blue-shifted, southeastern lobe of the flow (although additional red-shifted $\mathrm{H}_{2}$ knots were also discovered, B9 and B10, further to the northwest). Note that the southeastern jet in Fig. 6c corresponds to the blue-shifted MHEL peak, evident in the echelle data in Fig. 5 and discussed in the previous section.

The [FeII] $1.644 \mu \mathrm{m}$ line and a few of the stronger $(v=1-0)$ $\mathrm{H}_{2}$ lines can be seen in a spectrum (not shown) extracted from a region that encompasses the "SE jet". However, these emission lines are superimposed on to bright continuum and the weaker lines, needed for analysis of the excitation conditions in the MHEL region, were not observed with sufficient signal-tonoise. The $\mathrm{H}_{2} 1-0 \mathrm{~S}(1) / 2-1 \mathrm{~S}(1)$ ratio in the SE jet is at least 8, which is suggestive of shock-excitation or high-density fluorescence (discussed further below). The $\mathrm{H}_{2} 1-0 \mathrm{~S}(1) /[\mathrm{FeII}]$ line intensity ratio was found to be $\sim 4$. Unfortunately we do not have a good measure of $A_{\mathrm{v}}$ toward the SE jet. Although this does not influence the $\mathrm{H}_{2}$ ratio too severely, the $\mathrm{H}_{2} 1-0 \mathrm{~S}(1) /[\mathrm{FeII}]$ ratio could be as much as ten-times lower (for $A_{\mathrm{V}} \sim 30-40$ ). Even so, the ratio is "roughly" comparable to that measured for MHEL/FEL regions associated with low-mass YSOs (Davis et al. 2003).

The spectrum in Fig. 7, extracted from the IFU cube for the IRS 1 peak itself, shows the YSO's spectral energy distribution sharply-rising at longer wavelengths (as expected 


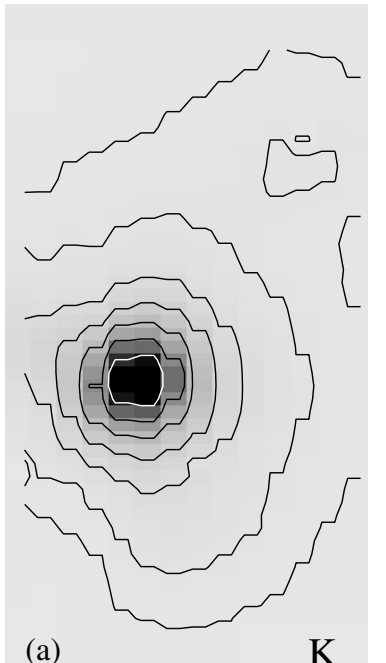

(a)

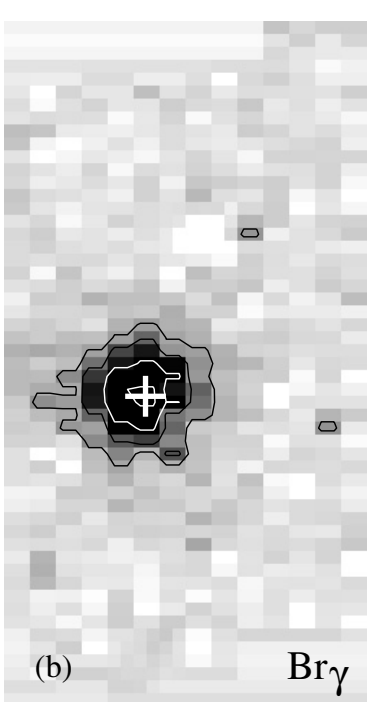

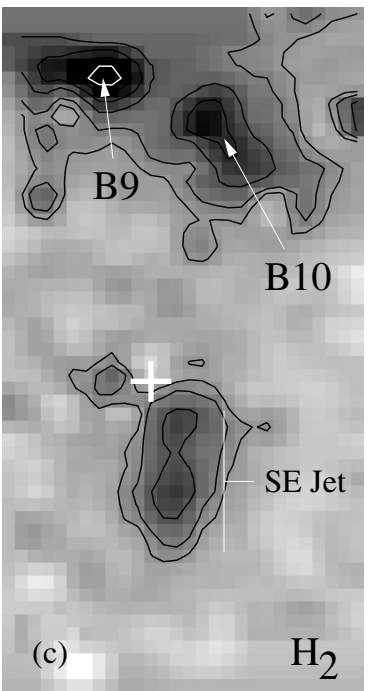

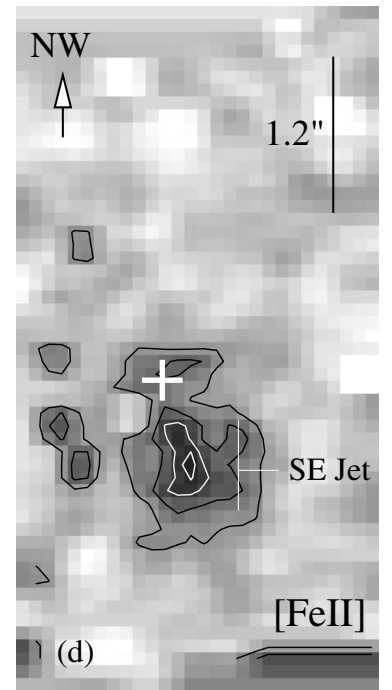

Fig. 6. Extracted IFU images of the bright IR source IRS 1. The $K$-band image a) has been extracted over a wavelength range $2.03-2.37 \mu \mathrm{m}$. The "narrow-band" images, in $\mathrm{Br} \gamma, \mathrm{H}_{2} 1-0 \mathrm{~S}(1)$ and [FeII] $1.644 \mu \mathrm{m}$ emission (b)-d)), have been extracted over 4-pixels ( 0.0031 $\left.\mu \mathrm{m}\right)$ in the dispersion direction, equivalent to twice the spectral resolution of the data. Adjacent continuum images (the average of images extracted at wavelengths red-ward and blue-ward of the emission line) have been subtracted from the three narrow-band images. Contours measure: a) 0.5 ,

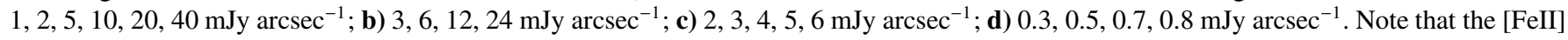
and $\mathrm{H}_{2}$ data have been binned over $2 \times 2$ pixels to improve signal-to-noise. In all images the pixel scale is $0.24^{\prime \prime} \times 0.12^{\prime \prime}$.

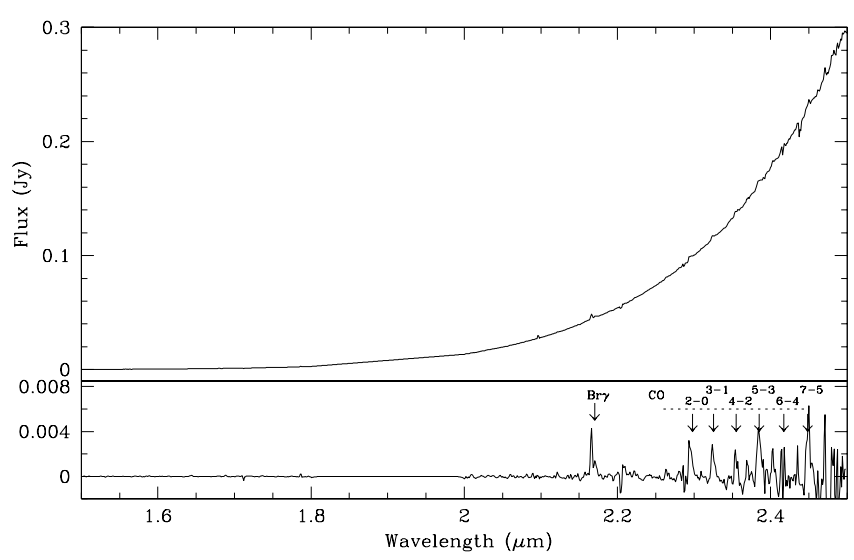

Fig. 7. HK spectrum toward the IRS 1 source, extracted over an areacentred on the $K$-band peak of IRS $1-$ of $0.72^{\prime \prime} \times 0.72^{\prime \prime}$. In the bottom plot the continuum has been fitted and removed to better show the line emission. Note that the increased noise at longer wavelengths is due to the strong flux and increased shot noise. In both spectra the gap between the $H$ and $K$-bands $(1.8-2.0 \mu \mathrm{m})$ where atmospheric transmission is poor, has been masked.

for an embedded source), plus the $\operatorname{Br} \gamma$ line emission noted above together with weak CO vibrational bands. The $\delta v=2$ band-heads between $v=2-0$ and $v=7-5$ are observed. The critical densities of these vibrational levels are $n_{\mathrm{H}} \sim$ $10^{12}-10^{13} \mathrm{~cm}^{-3}$ over the temperature range $2000-5000 \mathrm{~K}$. Like the $\mathrm{Br} \gamma$ emission, the $\mathrm{CO}$ is spatially confined to the IR peak and is also probably associated with (1) dense molecular gas heated by UV photons impinging on the inner disk surface (Najita et al. 1996b), or (2) the outer regions of accreting funnel-flows, where the gas is heated by adiabatic compression (Martin 1997).

For low-mass, classical T Tauri stars, Muzerolle et al. (1998) found a correlation between HI permitted emission-line fluxes and the accretion luminosity, the latter being derived separately from the infrared excess. Although this correlation has not yet been extended to embedded sources or massive YSOs, if IRAS 18151-1208 is associated with an accretion disk - which the presence of $\mathrm{Br} \gamma$ emission suggests is indeed the case - then the observed $\mathrm{Br} \gamma$ flux $\left(L_{\mathrm{Br} \gamma}=3.1 \times 10^{-18} \mathrm{~W} \mathrm{~m}^{-2}\right)$ may give at least a lower limit to the accretion luminosity, $L_{\text {acc }}$. If we assume an $A_{\mathrm{v}}$ of $30 \mathrm{mag}$ and a distance of $3 \mathrm{kpc}$, then the $\mathrm{Br} \gamma$ line flux yields an accretion luminosity of $\sim 120 L_{\odot}$. If the accretion luminosity is of the order of $10 \%$ of the bolometric luminosity (Muzerolle et al. 1998), then this in turn infers a luminosity of $\sim 10^{3} L_{\odot}$ for IRS 1 , a value which is typical of intermediate mass stars. However, this inferred luminosity is an order of magnitude lower than the bolometric luminosity of IRAS 18151-1208, a value derived from far-IR photometry by Sridharan et al. (2002) that is expected of high mass YSOs. This discrepancy can largely be explained by extinction and the flow inclination angle. $A_{\mathrm{v}}$ in particular is poorly known. $30 \mathrm{mag}$ of extinction towards the $\mathrm{Br} \gamma$ line-emission region is at best a lower limit, given that: (1) 20-30 mag of extinction is measured in the extended flow lobes (higher values are expected towards the embedded source); (2) the $\mathrm{Br} \gamma$ is thought to be excited very close to the central source, in accretion flows; and (3) the length of the flow and low radial velocities measured above suggest that the outflow is orientated close to the plane of the sky, so much of the accretion zone may be obscured by a circumstellar disk or torus.

\subsubsection{The $\mathrm{H}_{2}$ features in the extended flow lobes}

IFU observations of the main $\mathrm{H}_{2}$ shock features are shown in Fig. 8. Sample spectra, binned over small sections of each IFU field, illustrate the forest of $\mathrm{H}_{2}$ lines in the $H$ and $K$-bands. Notably, [FeII] emission was not detected in any of the 

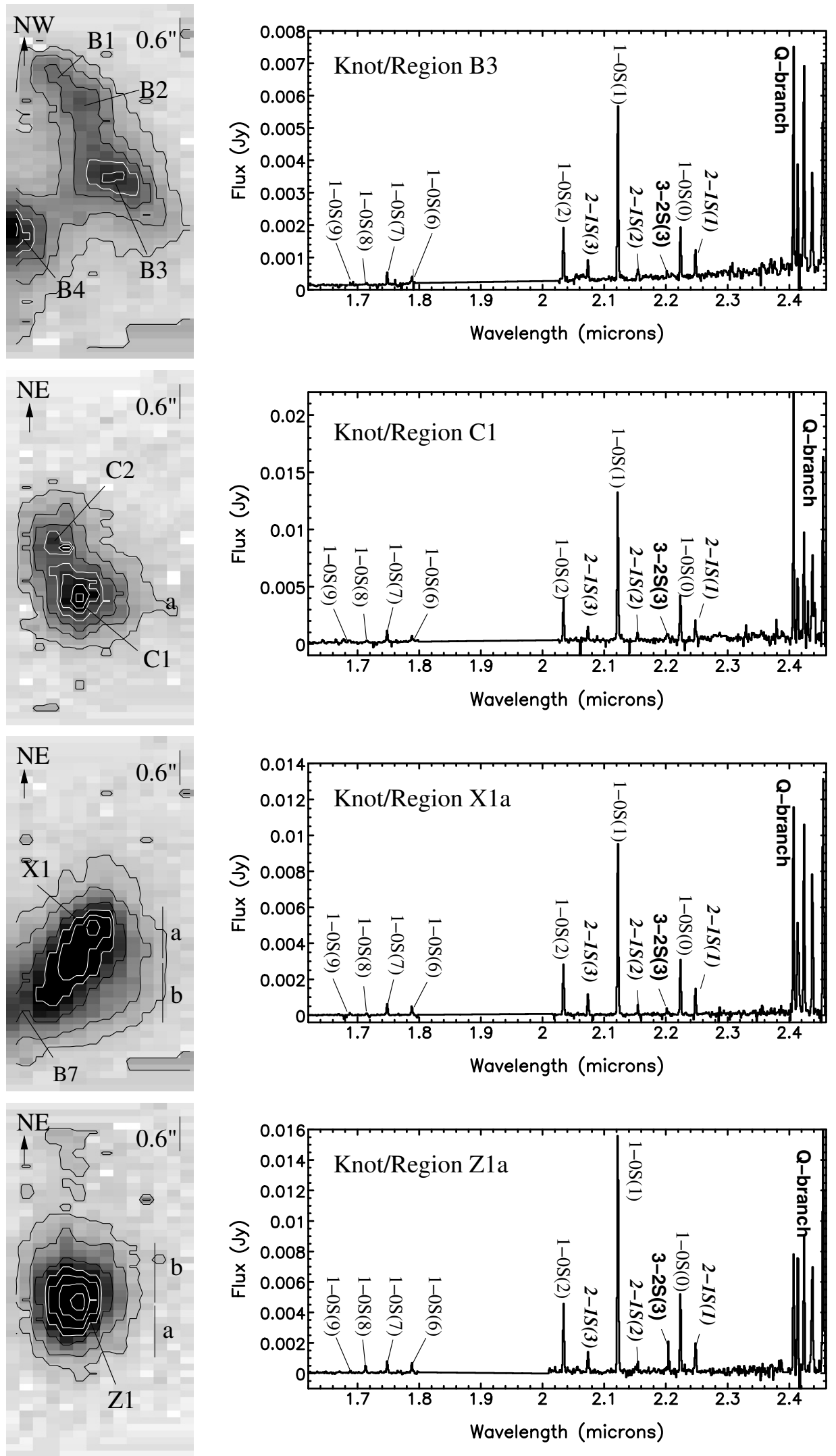

Fig. 8. IFU data toward the $\mathrm{H}_{2}$ emission-line regions $\mathrm{B} 1-\mathrm{B} 4$ and $\mathrm{C} 1-\mathrm{C} 2$ in the main northwest-southeast jet, and toward $\mathrm{X} 1$ and $\mathrm{Z} 1$ in the orthogonal northeast-southwest flow. Extracted $\mathrm{H}_{2} 1-0 \mathrm{~S}(1)$ images and sample spectra are shown in each case (described in Fig. 6 and Table 2). In each image the pixel scale is $0.24^{\prime \prime} \times 0.12^{\prime \prime}$; the contours measure $5,10,15,20,25,30,35 \mathrm{mJy} \operatorname{arcsec}^{-1}$ in the top two images, and $5,10,20,30,40,50,60 \mathrm{mJy} \operatorname{arcsec}^{-1}$ in the bottom two images. 

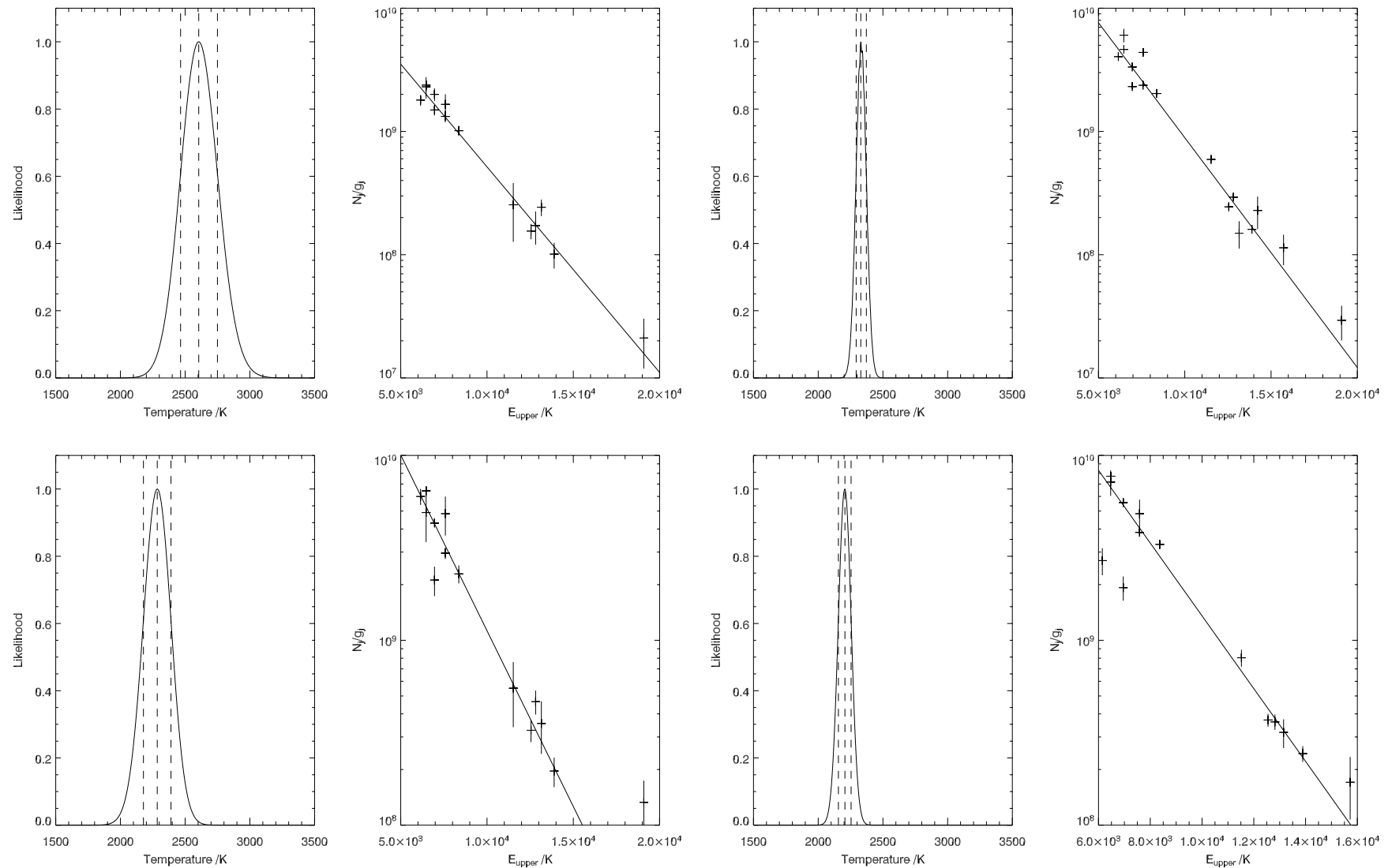

Fig. 9. Excitation diagrams (right) and plots of maximum likelihood (left) for four of the 10 regions for which spectra were extracted. The data are shown in Fig. 8. In the right-hand plots, the natural $\log$ of column density divided by the statistical weight, $N_{\mathrm{j}} / g_{\mathrm{j}}$, is plotted against the upper level energy. The error bars only reflect the goodness of the Gaussian fit to each emission line; they do not necessarily reflect the accuracy of telluric correction in regions between the $\mathrm{H}$ and $\mathrm{K}$ windows, and particularly for the Q-branch lines at 2.40-2.45 $\mu \mathrm{m}$. Similar diagrams were plotted for all 10 regions listed in Appendix A. To the left of each excitation diagram we also show plots of likelihood against excitation temperature, derived for each line column density measured in the region (see text for details). The vertical dashed lines denote the 1-sigma range in $T_{\mathrm{ex}}$ listed in Table 2.

observed features, which supports a low-excitation, molecular shock physics interpretation (extinction is unlikely to explain the lack of [FeII] emission for knots that are at some distance from IRS 1).

Spectra were extracted over two, three or four regions in each IFU field. These regions represent whole knots or parts of more extended knots (see Table 2 and Fig. 8 for details). In knots $\mathrm{X} 1$ and $\mathrm{Z} 1$, for example, a spectrum from the leading edge of each "bow shock" was extracted (X1a and Z1a) as well as from the flanks of each bow (X1b and Z1b). In all, spectra from 10 regions were extracted. $\mathrm{H}_{2}$ line intensities were measured and these are tabulated in Appendix A. The data were then plotted on excitation diagrams, examples of which are shown in Fig. 9.

By plotting the natural logarithm of the column density of the upper energy level, $N_{\mathrm{j}}$, divided by the statistical weight, $g_{\mathrm{j}}$, against the level energy, $E_{\mathrm{j}}$, an estimate of the gas excitation temperature, $T_{\mathrm{ex}}$, can be obtained, provided the energy level populations are close to thermal equilibrium (i.e. $N_{\mathrm{j}}=$ $4 \pi \lambda I_{\mathrm{j}} /\left(A_{\mathrm{j}} h c\right)$, where $I_{\mathrm{j}}$ is the line intensity and $A_{\mathrm{j}}$ is the Einstein coefficient; this in turn is proportional to the Boltzmann factor, $\left.\exp \left[-E_{\mathrm{j}} / k T_{\mathrm{ex}}\right]\right)$. Non-thermal excitation would yield different rotational and vibrational temperatures and the points in each diagram would lie on separate vibrational "ladders" (see, for example, the comparison between shocks and fluorescence in planetary nebulae given by Hora \& Latter 1994). Shock excitation produces points on a single line, or a curve if a range of gas temperatures is observed (the local slope of the curve then gives the excitation temperature for each transition).

In an excitation diagram, the random scatter of data points about the fit can be due to extinction (Nisini et al. 2002). By varying the extinction and maximising the goodness of each fit (maximising the correlation coefficient) we can estimate the extinction, $A_{\mathrm{v}}$, directly, without having to rely on dubious Q-branch intensities (the $1-0 \mathrm{~S}(1) / 1-0 \mathrm{Q}(3)$ ratio can be used to estimate $A_{\mathrm{v}}$, but the $1-0 \mathrm{Q}(3)$ line is in a poor region of the $K$ band and is therefore difficult to measure accurately). However, the fit to the data points remains good (above a $99 \%$ confidence level) for a relatively wide range in $A_{v}$, so although we do converge on an estimate for the extinction in each region, the errors on $A_{\mathrm{v}}$ are relatively high, of the order of $5 \mathrm{mag}$, because the actual "fit" is fairly insensitive to $A_{\mathrm{v}}$.

For each region considered in IRAS 18151-1208, the column densities, when plotted in an excitation diagram, were well represented by a linear fit (e.g. Fig. 9). A slightly better fit was produced with a second-order polynomial curve (not 
shown), although the resulting range in temperature was small in comparison to the mean value. Only for the single $v=3$ transition, with an upper energy of $19100 \mathrm{~K}$, does the curve appreciably deviate from the linear fit. This is not surprising given the relatively narrow range in level energies traced by the $v=1$ and $v=2$ lines; the pure rotational mid-infrared lines at a few hundred degrees Kelvin or the faint lines from the higher-excitation vibrational states with $v \geq 3$ or $J>10$ would almost certainly yield lower and higher excitation temperatures respectively (e.g. Burton \& Haas 1987). The fact that the points do not deviate appreciably from the fit in each region also points to a high degree of thermalisation for the $v=1$ and $v=2$ levels. The gas must be relatively dense $\left(n_{\mathrm{H} 2}>10^{4} \mathrm{~cm}^{-3}\right)$ in the post-shock region in each case.

A representative excitation temperature for the $v=1$ and $v=2$ transitions can be obtained from the linear fits. However, to better quantify the errors on $T_{\mathrm{ex}}$ we adopt a maximum likelihood method, which is described in Appendix B. The method allows us to use the error bars on the values of $N_{j} / g_{j}$ in the fit and to obtain a measurement of the uncertainty on the value of $T_{\text {ex }}$ which takes the uncertainty on the constant of proportionality into account.

Temperatures and extinction estimates for each region in IRAS 18151-1208 are listed in Table 2. Overall, the excitation conditions are typical of bow shocks and HH objects in jets and outflows from low-mass YSOs (e.g. Gredel 1994, 1996; Everett 1997; Eislöffel et al. 2000; Nisini et al. 2002; Lorenzetti et al. 2002). The extinction estimates are also not unreasonable. We can expect an $A_{\mathrm{v}}$ of at least a few towards IRAS 181511208, given its distance (the extinction through the galactic plane is typically of the order of 1.9 mag per kpc, although of course this value varies with direction; Sharov 1964). $A_{\mathrm{v}}$ is also expected to be high in this optically obscured, high-mass star forming region. The highest values are measured towards knots B1-B3. This is expected for these more distant features in the red-shifted flow lobe, where the jet is probably burrowing deeper into the ambient cloud. Changes in extinction between knots that are adjacent on the sky could be due to the ambient medium being clumpy (on arcsecond - or thousand-AU - size scales) or even local density enhancements produced by the individual shocks. However, the changes will also reflect the accuracy with which we can measure $A_{\mathrm{v}}$ from the excitation diagrams.

In Table 2 we also list $1-0 \mathrm{~S}(1) / 2-1 \mathrm{~S}(1)$ and $1-0 \mathrm{~S}(1) / 3$ $2 \mathrm{~S}(3)$ line ratios, as well as estimates of the ortho-para ratio. In conjunction with the excitation diagrams, these may be used to distinguish shock from fluorescent excitation.

The $\mathrm{H}_{2}$ 1-0 $\mathrm{S}(1) / 2-1 \mathrm{~S}(1)$ ratio alone is not a very robust discriminant: in photon-dominated regions (PDRs) at densities above $10^{5} \mathrm{~cm}^{-3}$ collisional de-excitation of far ultraviolet (FUV)-pumped molecules will thermalise the lower-energy states and yield line ratios of about 10-20, ratios typical of shocks or gas in LTE (Burton et al. 1990; Hollenbach \& Natta 1995). The higher-vibrational states are expected to retain a fluorescent population, however. The 1-0 S(1)/3-2 S(3) ratio will be $\sim 8$ in a dense PDR $\left(n_{\mathrm{H}}=10^{4}-10^{7} \mathrm{~cm}^{-3}\right.$, for a flux of $\sim 10^{3} G_{\mathrm{o}}$, where $G_{\mathrm{o}}$ is the average interstellar radiation field; Burton et al. 1990). Only for very high densities and very intense FUV radiation fields (FUV flux $\geq 10^{4} G_{\mathrm{o}}$ ) will this ratio approach a thermal or "shock" value of 10-100. Such conditions are only likely very close to the central star in a high-mass star forming region, and only then if the central YSO is sufficiently evolved (e.g. an Ultra-Compact HII region or later). The high values of the 1-0 S(1)/2-1 S(1) and 1-0 S(1)/3-2 S(3) ratios in Table 2 can therefore only be understood if the gas is shock-heated.

The ortho-para ratios for the $v=1$ states can be measured from the 1-0 S(0), 1-0 S(1) and 1-0 S(2) line intensities. $\mathrm{H}_{2}$ is formed on warm dust grains with a ratio $3: 1$, this being the ratio of the statistical weights of the nuclear spins (aligned:opposed spins). The ratio can be modified, however, in a sufficiently strong UV field because of the preferential pumping of the para states which have a higher opacity to FUV photons (Sternberg $\&$ Neufeld 1999). So although the overall ortho-para ratio may still be close to an equilibrium value of 3 , in a PDR the UVpumped molecules which contribute to the observed radiation may have a lower ratio. Indeed, ratios in the range 1.5-2.2 have been measured in some PDRs and planetary nebulae (e.g. Ramsay et al. 1993; Lumsden et al. 2001), especially for the higher-energy vibrational states $(v>1)$.

To calculate $\phi_{1}$, we adopt the technique described by Smith et al. (1997). The method benefits from the fact that the transitions are close in wavelength: $\phi_{1}$ is therefore relatively insensitive to extinction. The transitions also have upper states that are populated at essentially the same rotational excitation temperature. Measurements of $\phi_{1}$ should be relatively accurate, given the strength of the lines and their favourable positions within the $K$-band atmospheric window. Indeed, in IRAS 18151-1208 we see little variation from the thermal value of 3 ; the orthopara ratios for most of the knots in the region show little evidence for fluorescence.

Overall, in IRAS 18151-1208 the IFU data and our analysis of the gas excitation points to thermal excitation in shocks being the dominant mechanism, as is usually the case for molecular bow shocks and $\mathrm{HH}$ objects in outflows from low mass YSOs (e.g. Nisini et al. 2002; Smith et al. 2003).

\section{Discussion}

\subsection{The stellar population in the vicinity of IRAS 18151-1208}

JHK colour-colour and colour-magnitude diagrams are useful tools for identifying YSO candidates. Though our observations go deeper than the 2MASS observations and are of higher spatial resolution, we have broadband observations only at $\mathrm{K}$ $(2.2 \mu \mathrm{m})$ and the central object (IRS 1) is saturated in these data. We therefore utilise data from the 2MASS catalogue.

2MASS JHK magnitudes in a region centred on IRAS $18151-1208$, that is $2.3^{\prime} \times 2.3^{\prime}$ in size, similar to the field covered in our mosaics, were extracted from the SIMBAD database. Figure 10 (left) shows the stars plotted in a $(J-$ $H)-(H-K)$ colour-colour diagram. The thick curve shows the distribution of $(J-H)$ against $(H-K)$ for un-reddened main sequence stars, constructed from the infrared colours of Koornneef (1983). The lower end of the curve marks the early type stars, the upper end the late type stars. The two parallel 

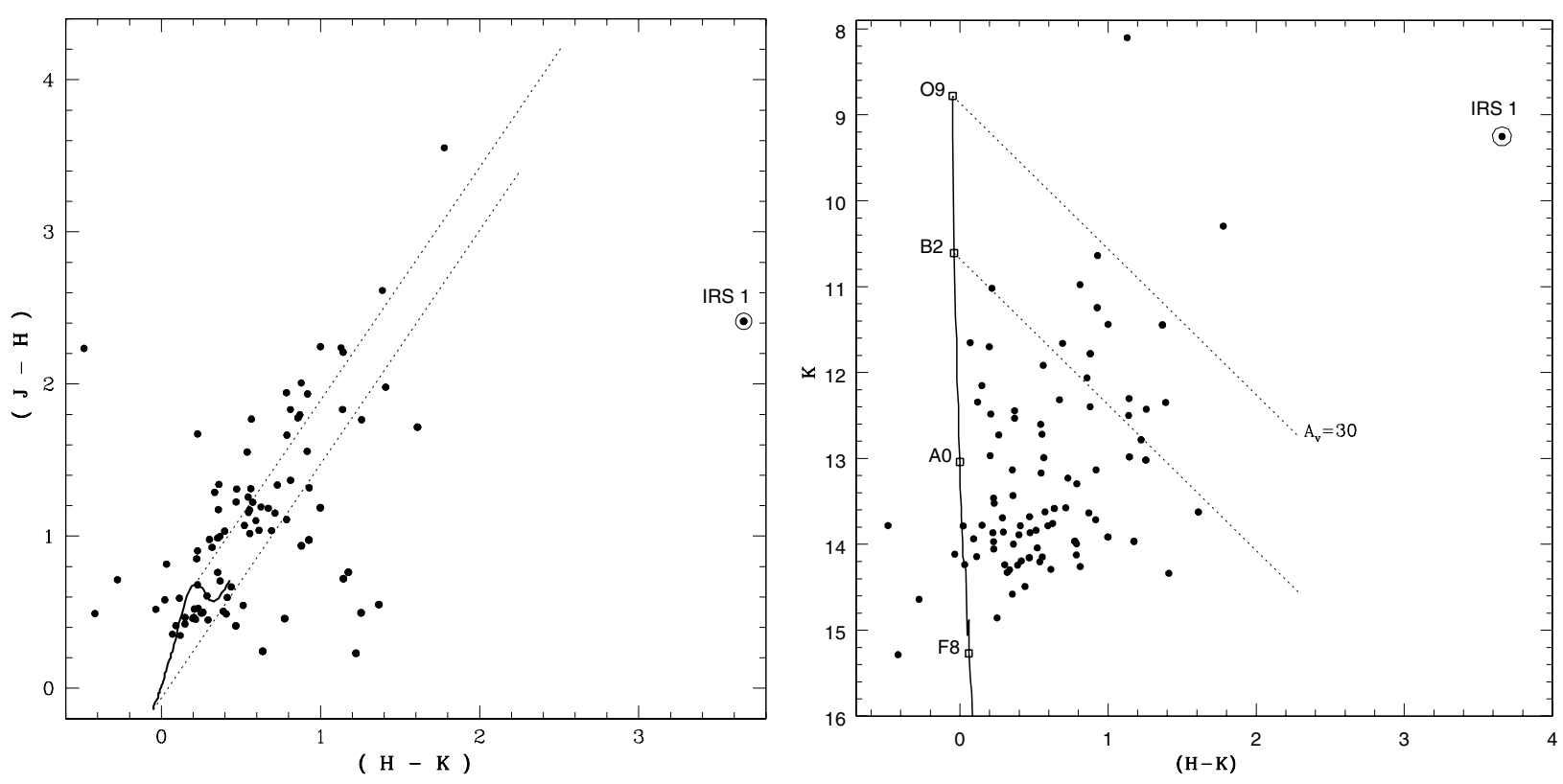

Fig. 10. Colour-colour and colour-magnitude diagrams derived from 2MASS photometry of stars in the vicinity of IRAS 18151-1208.

dotted lines in the colour-colour plot are the reddening vectors plotted up to $A_{\mathrm{v}}=30$ (reddening moves main-sequence stars along these vector toward the top-right of the diagram). We adopt a reddening law with $R_{\mathrm{V}}\left(A_{\mathrm{V}} / E_{(B-V)}\right)=5$, which is typical for dense clouds (Cardelli et al. 1989).

The stars within the reddening band - the region between the two parallel lines - are reddened main sequence stars seen along the line of sight. Figure 10 shows a large number of stars lying to the left of the reddening band. This region is typically occupied by evolved stars. However, at least some of the sources will be main sequence stars whose anomalous colours result from source confusion in the dense IRAS 18151-1208 cluster, specifically sources observed at different extinctions that are unresolved (or seen in projection) by 2MASS with its $2^{\prime \prime}$ pixel scale. The scatter in the colours of the main sequence stars about the reddening band is an indication of the accuracy with which 2MASS data can be used for population analysis in this dense, high-mass YSO cluster.

The region to the right of the reddening band should be occupied by YSOs. Some of these targets, particularly those to the far right in the diagram, will be embedded cluster members. However, by far the most conspicuously reddened star in Fig. 10 is IRS 1 , the dominant $K$-band source in IRAS 18151-1208. It has a large infrared excess and colours typical of luminous Class I objects (Lada \& Adams 1992).

In Fig. 10 (right) we also plot the $(H-K)$ colours of the same stars against their $K$ magnitudes. The continuous line marks the location of un-reddened main sequence stars (Koornneef 1983), from O9 down to F8. The dotted lines show the reddening vectors up to $A_{\mathrm{v}}=30$. IRS1 is again located far toward the top-right of the diagram. Its location in the colour-magnitude diagram is caused by intrinsic reddening and excess associated with the embedded YSO, rather than lineof-site reddening (e.g. Kumar et al. 2003). Thus, we are not able to accurately spectral type IRS 1 from this diagram alone.
Nevertheless, IRS 1 is clearly a luminous YSO. It is one of the brightest objects in our $K$-band image of the field, and is the most likely candidate for the source driving the A-B-C outflow.

\subsection{Are the molecular outflows driven by massive YSOs?}

The fact that the radial velocities observed in $\mathrm{H}_{2}$ are centred on the systemic velocity of IRAS 18151-1208, combined with the spatial coincidence of the $\mathrm{H}_{2}$ shocks with the high-velocity $\mathrm{CO}$ lobes in Fig. 2, indicates that the $\mathrm{H}_{2}$ and $\mathrm{CO}$ emission trace the same outflows. The $\mathrm{H}_{2}$ features are at the same kinematic distance as the massive YSO that dominates the FIR emission in IRAS 18151-1208. Also, our spectroscopy of the region around the source reveals emission at the very base of the jet, emission that connects the IRS 1 source with the more extended $\mathrm{H}_{2}$ features.

The rough spatial alignment of the $\mathrm{H}_{2}$ features suggests that they are part of two outflows, A-B-C and X-Y-Z. In the northwest-southeast flow the $\mathrm{H}_{2}$ features do not lie on a single, narrow axis. However, they can still be attributed to a single collimated flow. Observations of individual low-mass YSO outflows clearly show that $\mathrm{H}_{2}$ is preferentially excited in bow shock wings and along the walls of hollowed-out flow cavities (e.g. Davis \& Eislöffel 1995; Eislöffel 2000; Yun et al. 2001). This fact, combined with the short cooling times behind molecular shocks and the inhomogeneity of the ambient medium, can account for the often clumpy, unsymmetrical $\mathrm{H}_{2}$ line emission structures seen in near-IR images of outflows. This presumably explains the mis-alignment of the $\mathrm{H}_{2}$ features in the A-BC IRAS 18151-1208 outflow.

The overlapping blue- and red-shifted CO emission features in Beuther et al. (2002a)'s relatively low-resolution submillimetre map of IRAS 18151-1208 could be interpreted in terms of multiple, unresolved outflows. However, the higher 
resolution molecular images presented here indicate that the $\mathrm{CO}$ is probably associated with only two flows. Beuther et al. (2002a) attribute considerable mass and momentum to these two flows. From their survey they also derive a convincing correlation between the mechanical force from a $\mathrm{CO}$ flow and both the associated core dust mass and the source bolometric luminosity (effectively extending the correlations found for low mass YSOs by Lada 1995, and Bontemps et al. 1996). The CO outflows in IRAS 18151-1208 must therefore be associated with massive cores and luminous young stars, and because the $\mathrm{CO}$ flows are associated with the $\mathrm{H}_{2}$ jets, the collimated $\mathrm{H}_{2}$ jets must also be driven by massive YSOs.

Finally, the energy radiated in $\mathrm{H}_{2}$ is considerable, as one would expect for massive outflows from luminous young stars. The luminosity in all $\mathrm{H}_{2}$ lines, $L_{\mathrm{H} 2}$, is of course dependent on the distance to the source, as well as the extinction. Our echelle observations confirm that the $\mathrm{H}_{2}$ jets are at a kinematic distance of $\sim 3 \mathrm{kpc}$, and our IFU observations infer an extinction of the order of $20 \mathrm{mag}$. If the 1-0 S(1) flux represents a tenth of the total $\mathrm{H}_{2}$ ro-vibrational emission (Smith 1991), then from the narrow-band imaging we estimate the total $\mathrm{H}_{2}$ luminosity, corrected for $20 \mathrm{mag}$ of extinction $(\sim 2 \mathrm{mag}$ at $2.12 \mu \mathrm{m})$ to be $\sim 0.7 L_{\odot}$ for the main A-B-C jet and $0.05 L_{\odot}$ for the orthogonal $\mathrm{X}-\mathrm{Y}-\mathrm{Z}$ jet. Both values are high in comparison to $\mathrm{H}_{2}$ luminosities for low-mass flows (e.g. Davis et al. 1997), by 1-3 orders of magnitude. They are also comparable to the mechanical luminosity of the CO outflow (Beuther et al. 2002a, report a value of $1.1 L_{\odot}$ for $L_{\mathrm{CO}}$ ). The energy radiated by $\mathrm{H}_{2}$, or more precisely by all molecular coolants, should be comparable to the mechanical luminosity in the $\mathrm{CO}$ flow if the molecular flow is swept-up and entrained by a collimated jet in a momentumconserving fashion. This is widely believed to be the case for low-mass flows; it also seems to be the case here for this highmass system.

\subsection{Flow collimation}

To date most molecular outflows from massive YSOs have been studied at (sub)mm wavelengths, often with single-dish telescopes and therefore at low spatial resolutions (e.g. Shepherd \& Churchwell 1996; Ridge \& Moore 2001). This is perhaps not surprising given the increased extinction and greater distances associated with massive YSOs. These data were initially seen as indicating that flow collimation is poorer in massive YSO outflows than it is in flows from low mass protostars. In their review, Richer et al. (2000) suggest that massive flows have length-to-width ratios of no more than 1.8, although Beuther et al. (2002a) recently claimed collimation factors of $>2$ in a number of high mass YSO flows. These values compare with collimation factors on the order of 1-10 in low-mass YSO flows.

However, recent higher-resolution interferometric radio maps of a few massive star forming regions have resolved seemingly poorly collimated flows into a number of adjacent, overlapping "jets" (Beuther et al. 2002c; Beuther et al. 2003; $\mathrm{Su}$ et al. 2004). In IRAS $05358+3543$, for example, at least three CO flows have been detected, one of which exhibits a collimation factor of $\sim 10$, the highest collimation factor so far observed in a massive star-forming region, and a number which is certainly as high as that typically seen in low-mass star forming regions.

Our survey of near-IR $\mathrm{H}_{2}$ jets (Varricatt et al. 2004) was aimed at searching for collimated flows in sites of massive star formation. Initial results support the idea that there are multiple, collimated flows associated with many regions, even though in some cases the extinction to the region hampers our observations at $2.2 \mu \mathrm{m}$. Where circumstance allows (i.e. for sources situated near the front side of a cloud, or for outflows breaking out of a cloud) we do find evidence for "HH-type" knots and bow shocks. In many of the flows observed, such features have been discovered which, in some cases, suggest the presence of well-collimated jets.

IRAS 18151-1208 is one of the more spectacular examples from the survey. Based on the approximate overall diameter of the "bow cluster" B1-B4 ( 14000 AU) and its distance from IRS 1 ( $~ 80000 \mathrm{AU})$ we estimate a collimation factor of $\sim 6$. If we use the fainter, more distant knot A we would estimate a collimation factor about twice as large. Similar factors can of course be inferred from the images of the orthogonal X-Y-Z jet.

How do these numbers compare with $\mathrm{H}_{2}$ observations of jets from low-mass YSOs? Many low mass flows have been shown to change direction or "wiggle". Also, $\mathrm{H}_{2}$ emission features will be short-lived if the underlying jet is switched off (or re-directed), the cooling time behind a molecular shock being only about a year. So a direct comparison is difficult. However, if we consider the "archetypal" molecular hydrogen jet, HH 212 (Zinnecker et al. 1998), we do see many similarities. HH 212 is powered by a $15 L_{\odot}$ Class I source. Both lobes of the jet are traced by a string of compact, 500 AU-sized knots. The knots mark the lobes of the jet out to a distance of about $10000 \mathrm{AU}$ from the source. At a distance of $20000 \mathrm{AU}$, slightly larger bows are evident, while at distances of 30000-35000 AU, huge sweeping bows are observed. These much larger bow shocks (NB3 and SB3) may be the counterparts of the features we label B1-B4 in IRAS 18151-1208. This being the case, then the "collimation factor" in HH 212, based on the diameter of the large bows NB3 and SB3, is essentially the same as that measured here for the northwestern lobe of the IRAS 18151-1208 flow. In morphological terms the two flows are quite similar, even though the distance between IRS 1 and B1-B4 in IRAS 18151-1208 is about twice the distance between the $\mathrm{HH} 212$ source and NB3/SB3. It is also worth noting that the inner knots in HH 212 suggest a far higher collimation factor. It is unlikely that the HH 212 flow suddenly expands between the last of the inner compact knots and the larger bows NB3 and SB3. Instead, the sweeping bows NB3 and SB3 are probably driven by a highly collimated flow, or at least a flow with a very steep radial density gradient (the jet may be accompanied by a wide-angle, unobserved wind). In a similar fashion, the underlying jet in IRAS 18151-1208 that powers the knots labeled B, C and A could also be far more collimated than is suggested by the collimation factor derived from the size of the bow cluster B1-B4, above. 


\section{Conclusions}

The outflow activity associated with the luminous young star IRAS 18151-1208 is examined via near-infrared imaging and both high-spectral-resolution echelle and intermediatespectral-resolution integral field spectroscopy. We arrive at the following conclusions:

1. Two collimated flows, denoted the A-B-C and X-Y-Z jets, are evident in our images. The axis of each flow is delineated by bright bow shocks seen here in $\mathrm{H}_{2}$ 1-0 $\mathrm{S}(1)$ emission. Like their low-mass counterparts the $\mathrm{H}_{2}$ bows are closely associated with bipolar molecular $(\mathrm{CO})$ outflows.

2. The $\mathrm{H}_{2}$ features suggest a high degree of collimation in each outflow. In the main northwest-southeast A-B-C flow, a collimation factor of at least 6 is measured.

3. Although on large scales the northwestern, red-shifted flow lobe in the A-B-C jet is brightest in $\mathrm{H}_{2}$, within 1-2" (3000-6000 AU) of the IRS 1 source the emission is predominantly blue-shifted. Like MHEL regions associated with low-mass YSOs, in IRAS 18151-1208 the $\mathrm{H}_{2}$ at the jet base is blue-shifted by a few $\mathrm{km} \mathrm{s}^{-1}$, and the emission profile is narrow. The emission peak is offset by less than an arcsecond along the jet axis and, again like its lowmass counterparts, is accompanied by [FeII] forbidden line emission.

4. Our excitation analysis suggests that the $\mathrm{H}_{2}$ in the extended flow lobes and bow shocks is excited in low-excitation molecular shocks. Like molecular bows in low mass YSO outflows, there is little evidence of fluorescent excitation in the near-IR data. C-shocks are preferred over J-type shocks, because of the lack of [FeII] in the extended flow, and because of the wide $\mathrm{H}_{2}$ line profiles observed throughout the region.

The features labeled X-Y-Z are probably associated with a younger, more deeply embedded source than the main A-B$\mathrm{C}$ jet (which we assume is driven by IRS 1). Even so, we find that - qualitatively and quantitatively - both outflows are very similar to their low-mass counterparts, flows that are driven by accretion on to Class 0 and Class I solar-mass stars. Thus, the observations presented here favour a disk-accretion star formation mechanism that is essentially a scaled-up version of that proposed for low mass young stars.

Acknowledgements. We would like to thank Henrik Beuther for kindly and very promptly supplying his (sub)mm data, which we have reproduced here in Fig. 2, and Brunella Nisini for her comments as referee. We acknowledge the data reduction facilities provided by the Starlink project, which is run by the CCLRC on behalf of PPARC. The United Kingdom Infrared Telescope is operated by the Joint Astronomy Centre on behalf of the UK Particle Physics and Astronomy Research Council. Some of the data reported here were obtained as part of the UKIRT Service Observing Programme. We have made use of 2MASS data obtained as part of the Two Micron All Sky Survey (2MASS), a joint project of the University of Massachusetts and the Infrared Processing and Analysis Center/California Institute of Technology, funded by the National Aeronautics and Space Administration and the National Science Foundation. This research has also made use of the SIMBAD database, operated at CDS, Strasbourg, France.

\section{References}

Ayala, S., Curiel, S., Raga, A. C., Noriega-Crespo, A., \& Salas, L. 1998, A\&A, 332, 1055

Beuther, H., Schilke, P., Sridharan, T. K., et al. 2002a, A\&A, 383, 892

Beuther, H., Walsh, A., Schilke, P., et al. 2002b, A\&A, 390, 289

Beuther, H., Walsh, A., Schilke, P., et al. 2002c, A\&A, 387, 931

Beuther, H., Schilke, P., \& Stanke, T. 2003, A\&A, 408, 601

Blondin, J. M., Königl, A., \& Fryxell, B. A. 1989, 337, L37

Bontemps, S., Andre, P., Terebey, S., \& Cabrit, S. 1996, A\&A, 311, 858

Bragg, S. L., Smith, W. H., \& Brault, J. W. 1982, ApJ, 263, 994

Brand, J., \& Blitz, L. 1993, A\&A, 275, 67

Bronfman, L., Nyman, L. A., \& May, J. 1996, A\&AS, 115, 81

Brooks, K. J., Garay, G., Mardones, D., \& Bronfman, L. 2003, ApJ, 594, L131

Burton, M. G., \& Haas, M. R. 1997, A\&A, 327, 309

Burton, M. G., Hollenbach, D. J., \& Tielens, A. G. G. M. 1990, ApJ, 365,620

Cabrit, S., Ferreira, J., \& Raga, A. C. 1999, A\&A, 343, L61

Cardelli, J. A., Clayton, G. C., \& Mathis, J. S. 1989, ApJ, 345, 245

Churchwell, E. 2002, in Hot Star Workshop III: The Earliest Stages of Massive Star Birth, ed P. A. Crowther, ASP Conf. Proc., 267, 3

Davis, C. J., \& Eislöffel, J. 1995, A\&A, 300, 851 (Erratum: A\&A, 305, 694)

Davis, C. J., Ray, T. P., Eislöffel, J., \& Corcoran, D. 1997, A\&A, 324, 263

Davis, C. J., Moriarty-Schieven, G. H., Eislöffel, J., Hoare, M. G., \& Ray, T. P. 1998, AJ, 115, 1118

Davis, C. J., Hodapp, K. W., \& Desroches, L. 2001a, A\&A, 377, 285

Davis, C. J., Ray, T. P., Desroches, L., \& Aspin, C. 2001b, MNRAS, 326, 524

Davis, C. J., Whelan, E., Ray, T. P., \& Chrysostomou, A. 2003, A\&A, 397, 693

Downes, T. P., \& Ray, T. P. 1999, A\&A, 345, 977

Eislöffel, J. 2000, A\&A, 354, 236

Eislöffel, J., Smith, M. D., \& Davis, C. J. 2000, A\&A, 359, 1147

Everett, M. E. 1997, 478, 246

Folha, D. F. M., \& Emerson, J. P. 2000, A\&A, 365, 90

Garcia, P. V. J., Ferreira, J., Cabrit, S., \& Binette, L. 2001, A\&A, 377, 589

Gredel, R. 1994, A\&A, 292, 580

Gredel, R. 1996, A\&A, 305, 582

Hartigan, P., Raymond, J. C., \& Hartmann, L. 1987, ApJ, 316, 323

Hollenbach, D., \& Natta, A. 1995, ApJ, 455, L33

Hora, J. L., \& Latter, W. B. 1994, ApJ, 437, 281

Koornneef, J. 1983, A\&A, 128, 84

Kumar, M. S. N., Bachiller, R., \& Davis, C. J. 2002, ApJ, 576, 313

Kumar, M. S. N., Ojha, D. K., \& Davis, C. J. 2003, ApJ, 598, 1107

Lada, C. J. 1985, ARA\&A, 23, 267

Lada, C. J., \& Adams, F. C. 1992, ApJ, 393, 278

Lee, C. F., Mundy, L. G., Reipurth, B., Ostriker, E. C., \& Stone, J. M. 2000, ApJ, 542, 925

Lorenzetti, D., Gianninni, T., Vitali, F., Massi, F., \& Nisini, B. 2002, ApJ, 564, 839

Lumsden, S. L., Puxley, P. J., \& Hoare, M. G. 2001, MNRAS, 328, 419

Martin, S. C. 1997, ApJ, 478, L33

Mountain, C. M., Robertson, D., Lee, T. J., \& Wade, R. 1990, in Instrumentation in Astronomy, SPIE v1235, VII, 25

Muzerolle, J., Hartmann, L., \& Calvet, N. 1998, AJ, 116, 2965

Najita, J., Carr, J. S., \& Tokunaga, A. T. 1996a, ApJ, 456, 292

Najita, J., Carr, J. S., Glassgold, A. E., Shu, F. H., \& Tokunaga, A. T. 1996b, ApJ, 462, 919 
Nisini, B., Caratti o Garatti, A., Gianninni, T., \& Lorenzetti, D. 2002, A\&A, 393, 1035

Oliva, E., \& Origlia, L. 1992, A\&A, 254, 466

Poras, A., Cruz-González, I., \& Salas, L. 2000, A\&A, 361, 660

Pyo, T. S., Hayashi, M., Kobayashi, N., et al. 2002, ApJ, 570, 724

Raga, A. C. 1993, ApS\&S, 208, 163

Ramsay, S. K., Chrysostomou, A., Geballe, T. R., Brand, P. W. J. L., \& Mountain, M. 1993, MNRAS, 263, 695

Ramsay Howat, S. K., Ellis, M. A., Gostick, D. C., et al. 2000, SPIE, 4008, 1067

Richer, J., Shepherd, D., Cabrit, S., Bachiller, R., \& Churchwell, E. 2000, in Protostars \& Planets IV, ed. V. Mannings, A. P. Boss, \& S. S. Russell (Tucson: University of Arizona Press), 867

Ridge, N., \& Moore, T. J. 2001, A\&A, 378, 495

Roche, P. F., et al. 2002, in Instrument Design and Performance for Optical/IR Ground-Based Telescopes, ed. M. Iye, \& A. F. Moorwood, SPIE, 4841, 901

Safier, P. N. 1993, ApJ, 408, 115

Shang, H., Glassgold, A. E., Shu, F. H., \& Lizano, S. 2002, ApJ, 564, 853

Sharov, A. S. 1964, SvA, 7, 689

Shepherd, D. S., \& Churchwell, E. 1996, 472, 225
Shepherd, D. S., Yu, K. C., Bally, J., \& Testi, L. 2000, ApJ, 535, 833

Smith, M. D. 1991, MNRAS, 252, 378

Smith, M. D., Davis, C. J., \& Lioure, A. 1997, A\&A, 327, 1206

Smith, M. D., Khanzadyan, T., \& Davis, C. J. 2003, MNRAS, 339, 524

Sridharan, T. K., Beuther, H., Schilke, P., Menten, K. M., \& Wyrowski, F. 2002, ApJ, 566, 931

Sternberg, A., \& Neufeld, D. A. 1999, ApJ, 516, 371

Su, Y.-N., Zhang, Q., \& Lim, J. 2004, ApJ, in press

Takami, M., Chrysostomou, A., Ray, T. P., et al. 2004, A\&A, 416, 213

Todd, S. P., Wells, M., Ramsay Howat, S. K., \& Hastings, P. R. 2003, SPIE, 4842, 151

Varricatt, W. P., Davis, C. J., Ramsay Howatt, S. K., \& Todd, S. P. 2004 , in preparation

Völker, R., Smith, M. D., Suttner, G., \& Yorke, H. W. 1999, A\&A, 343,595

Williams, S. J., Fuller, G. A., \& Sridharan, T. K. 2004, A\&A, in press Wood, D. O. S., \& Churchwell, E. 1999, ApJ, 340, 265

Yun, J. L., Santos, C. A., Clemens, D. P., et al. 2001, A\&A, 372, L33

Zinnecker, H., McCaughrean, M. J., \& Rayner, J. 1998, Nature, 394, 27 


\section{Online Material}




\section{Appendix A: Measured line intensities}

Below we tabulate the line intensities measured for each region observed with the IFU. In some cases, spectra for two or more adjacent regions were extracted from the data, so that a comparison could be made, for example, between the gas at the head of and in the flanks of a bow shock. Note, however, that although fluxes were calibrated, these data were observed in thin and/or variable cirrus, so while line ratios should be accurate, absolute flux densities may be accurate only to within a factor of two or three.

\section{Appendix B: Maximum Likelihood method for deriving $T_{\mathrm{ex}}$}

Maximum likelihood methods are based on Bayes theorem, which states that:

$\operatorname{Prob}(X \mid Y, I)=\frac{\operatorname{Prob}(Y \mid X, I) \times \operatorname{Prob}(X \mid I)}{\operatorname{Prob}(Y \mid I)}$.

This can be used to determine the likelihood of a model, $X$, given the measured data, $Y$, and prior knowledge of the likelihood of the model, $I$. For the purpose of comparing the relative likelihood of a number of models $\operatorname{Prob}(Y \mid I)$ can be ignored as a normalisation factor. If we assume that all the models that we are to compare are previously thought to be equally likely, then $I$ is a uniform prior, and $\operatorname{Prob}(X \mid I)$ is simply a constant (given a reasonable amount of data the final fit should not be significantly sensitive to any sensible prior function that is chosen). In this case the equation reduces to

$\operatorname{Prob}(X \mid Y) \propto \operatorname{Prob}(Y \mid X)$.

In other words, the likelihood that a given model describes the system from which the data are obtained is proportional to the probability of obtaining that set of data if that were the correct model.

For the excitation data presented in this paper, the Boltzmann distribution was fitted to the measured column densities using a maximum likelihood method to determine the most likely values of $T_{\mathrm{ex}}$ and the constant of proportionality:

$\frac{N_{j}}{g_{j}}=\exp \left(\frac{-E_{j}}{k T_{\mathrm{ex}}}+\alpha\right)$

where the constant of proportionality has been written as $e^{\alpha}$.

The relative likelihood of a range of values of $T_{\mathrm{ex}}$ and $\alpha$ was calculated for each measured line flux by regarding each combination of $T_{\mathrm{ex}}$ and $\alpha$ as an alternative model. The probability of obtaining the data given each model, and hence the relative likelihood of the model given the data, was calculated by assuming a Gaussian error distribution on the measurements of the line intensity:

$L_{j}\left(T_{\mathrm{ex}}, \alpha\right) \propto \frac{\exp \left[-(\delta / 2 \sigma)^{2}\right]}{\sigma \sqrt{2 \pi}}$,

where $\delta$ is the difference between the value of $N_{j} / g_{j}$ derived from the measured intensity and that calculated using Eq. (B.3) (the model). $\sigma$ is the $1 \sigma$ error on the measured value of $N_{j} / g_{j}$.
The combined likelihood of the parameters using all the line fluxes is calculated by taking the product of all the individual likelihoods. The likelihood of each value of $T_{\mathrm{ex}}$, taking into account the uncertainty in $\alpha$, can be calculated by marginalising the likelihood, or integrating over all values of $\alpha$ to produce a one dimensional likelihood curve as a function of $T_{\mathrm{ex}}$ :

$L\left(T_{\mathrm{ex}}\right)=\int_{-\infty}^{\infty}\left(\prod_{j} L_{j}\left(T_{\mathrm{ex}}, \alpha\right)\right) \mathrm{d} \alpha$.

The most likely value of $T_{\mathrm{ex}}$ is that which maximises $L\left(T_{\mathrm{ex}}\right)$. The $68 \%$ confidence levels (the narrowest possible range of values of $T_{\mathrm{ex}}$ to include $68 \%$ of the total area under the likelihood curve) were measured as an equivalent of $1 \sigma$ error bars on a Gaussian distribution. 
C. J. Davis et al.: Collimated molecular jets from high-mass young stars: IRAS 18151-1208, Online Material p 3

Table A.1. $\mathrm{H}_{2}$ line intensities.

\begin{tabular}{ccccccc}
\hline \hline Line & $\lambda$ & $B 1$ & $B 2$ & $B 3$ & $B 4$ & $B 7$ \\
\hline $1-0 S(0)$ & 2.2235 & $0.16 \mathrm{e}-5(0.6 \mathrm{e}-6)$ & $0.34 \mathrm{e}-5(0.3 \mathrm{e}-6)$ & $3.70 \mathrm{e}-6(3.0 \mathrm{e}-7)$ & $0.32 \mathrm{e}-5(0.6 \mathrm{e}-6)$ & $0.64 \mathrm{e}-5(0.3 \mathrm{e}-6)$ \\
$1-0 S(1)$ & 2.1218 & $0.53 \mathrm{e}-5(0.2 \mathrm{e}-6)$ & $0.11 \mathrm{e}-4(0.2 \mathrm{e}-6)$ & $1.44 \mathrm{e}-5(1.7 \mathrm{e}-6)$ & $0.11 \mathrm{e}-4(0.6 \mathrm{e}-6)$ & $0.24 \mathrm{e}-4(0.3 \mathrm{e}-6)$ \\
$1-0 S(2)$ & 2.0338 & $0.14 \mathrm{e}-5(0.2 \mathrm{e}-6)$ & $0.30 \mathrm{e}-5(0.2 \mathrm{e}-6)$ & $3.80 \mathrm{e}-6(4.0 \mathrm{e}-7)$ & $0.29 \mathrm{e}-5(0.6 \mathrm{e}-6)$ & $0.69 \mathrm{e}-5(0.3 \mathrm{e}-6)$ \\
$1-0 S(6)$ & 1.7880 & $<0.3 \mathrm{e}-6$ & $0.50 \mathrm{e}-6(0.2 \mathrm{e}-6)$ & $0.60 \mathrm{e}-6(3.0 \mathrm{e}-7)$ & $0.60 \mathrm{e}-6(0.1 \mathrm{e}-6)$ & $0.14 \mathrm{e}-5(0.6 \mathrm{e}-6)$ \\
$1-0 S(7)$ & 1.7480 & $0.44 \mathrm{e}-6(0.1 \mathrm{e}-6)$ & $0.70 \mathrm{e}-6(1.0 \mathrm{e}-7)$ & $1.00 \mathrm{e}-6(3.0 \mathrm{e}-7)$ & $0.78 \mathrm{e}-6(0.1 \mathrm{e}-6)$ & $0.18 \mathrm{e}-5(0.6 \mathrm{e}-6)$ \\
$1-0 S(8)$ & 1.7147 & $<0.2 \mathrm{e}-6$ & $<0.2 \mathrm{e}-6$ & $<0.3 \mathrm{e}-6$ & $<0.4 \mathrm{e}-6$ & $<0.5 \mathrm{e}-6$ \\
$1-0 S(9)$ & 1.6877 & $<0.2 \mathrm{e}-6$ & $0.73 \mathrm{e}-6(1.0 \mathrm{e}-7)$ & $<0.3 \mathrm{e}-6$ & $<0.4 \mathrm{e}-6$ & $0.47 \mathrm{e}-6(0.3 \mathrm{e}-6)$ \\
$2-1 S(1)$ & 2.2477 & $0.98 \mathrm{e}-6(0.6 \mathrm{e}-6)$ & $0.24 \mathrm{e}-5(0.3 \mathrm{e}-6)$ & $2.10 \mathrm{e}-6(3.0 \mathrm{e}-7)$ & $0.15 \mathrm{e}-5(0.6 \mathrm{e}-6)$ & $0.35 \mathrm{e}-5(0.3 \mathrm{e}-6)$ \\
$2-1 S(2)$ & 2.1542 & $0.25 \mathrm{e}-6(0.2 \mathrm{e}-6)$ & $0.11 \mathrm{e}-5(0.3 \mathrm{e}-6)$ & $0.13 \mathrm{e}-5(0.2 \mathrm{e}-6)$ & $0.43 \mathrm{e}-6(0.5 \mathrm{e}-6)$ & $<0.1 \mathrm{e}-5$ \\
$2-1 S(3)$ & 2.0735 & $0.48 \mathrm{e}-6(0.2 \mathrm{e}-6)$ & $0.13 \mathrm{e}-5(0.2 \mathrm{e}-6)$ & $1.70 \mathrm{e}-6(4.0 \mathrm{e}-7)$ & $0.95 \mathrm{e}-6(0.5 \mathrm{e}-6)$ & $0.29 \mathrm{e}-5(0.3 \mathrm{e}-6)$ \\
$3-2 S(3)$ & 2.2014 & $0.87 \mathrm{e}-6(0.6 \mathrm{e}-6)$ & $0.75 \mathrm{e}-6(0.4 \mathrm{e}-6)$ & $4.60 \mathrm{e}-7(2.0 \mathrm{e}-7)$ & $<0.4 \mathrm{e}-6$ & $0.56 \mathrm{e}-6(0.3 \mathrm{e}-6)$ \\
$1-0 Q(1)$ & 2.4066 & $0.77 \mathrm{e}-5(0.5 \mathrm{e}-6)$ & $0.17 \mathrm{e}-4(0.8 \mathrm{e}-6)$ & $1.20 \mathrm{e}-5(1.2 \mathrm{e}-6)$ & $0.19 \mathrm{e}-4(0.1 \mathrm{e}-5)$ & $0.30 \mathrm{e}-4(0.2 \mathrm{e}-5)$ \\
$1-0 Q(2)$ & 2.4134 & $0.30 \mathrm{e}-5(0.6 \mathrm{e}-6)$ & $0.62 \mathrm{e}-5(0.8 \mathrm{e}-6)$ & $6.10 \mathrm{e}-6(1.2 \mathrm{e}-6)$ & $0.65 \mathrm{e}-5(0.1 \mathrm{e}-5)$ & $0.12 \mathrm{e}-4(0.2 \mathrm{e}-5)$ \\
$1-0 Q(3)$ & 2.4237 & $0.75 \mathrm{e}-5(0.5 \mathrm{e}-6)$ & $0.15 \mathrm{e}-4(0.8 \mathrm{e}-6)$ & $1.55 \mathrm{e}-5(1.5 \mathrm{e}-6)$ & $0.16 \mathrm{e}-4(0.1 \mathrm{e}-5)$ & $0.29 \mathrm{e}-4(0.2 \mathrm{e}-5)$ \\
$1-0 Q(4)$ & 2.4375 & $0.30 \mathrm{e}-5(0.5 \mathrm{e}-6)$ & $0.76 \mathrm{e}-5(0.9 \mathrm{e}-6)$ & $7.20 \mathrm{e}-6(1.5 \mathrm{e}-6)$ & $0.58 \mathrm{e}-5(0.1 \mathrm{e}-5)$ & $0.12 \mathrm{e}-4(0.2 \mathrm{e}-5)$ \\
$1-0 Q(5)$ & 2.4548 & $0.72 \mathrm{e}-5(0.6 \mathrm{e}-6)$ & $0.13 \mathrm{e}-4(0.8 \mathrm{e}-6)$ & $1.60 \mathrm{e}-5(1.5 \mathrm{e}-6)$ & $0.98 \mathrm{e}-5(0.1 \mathrm{e}-5)$ & $0.30 \mathrm{e}-4(0.2 \mathrm{e}-5)$ \\
\hline
\end{tabular}

Line intensities given in $\mathrm{Jy} \mu \mathrm{m}$ (to convert to $\mathrm{W} \mathrm{m}^{-2}$ multiply by $3 \times 10^{-24} / \lambda^{2}$ ). Errors are given in brackets. Vacuum wavelengths in column two are in microns.

Table A.2. $\mathrm{H}_{2}$ line intensities.

\begin{tabular}{ccccccc}
\hline \hline Line & $\lambda$ & $C 1$ & $X 1 a$ & $X 1 b$ & $Z 1 a$ & $Z 1 b$ \\
\hline $1-0 S(0)$ & 2.2235 & $0.10 \mathrm{e}-4(0.6 \mathrm{e}-6)$ & $0.72 \mathrm{e}-5(0.2 \mathrm{e}-6)$ & $0.76 \mathrm{e}-5(0.3 \mathrm{e}-6)$ & $0.12 \mathrm{e}-4(0.3 \mathrm{e}-6)$ & $0.12 \mathrm{e}-4(0.3 \mathrm{e}-6)$ \\
$1-0 S(1)$ & 2.1218 & $0.31 \mathrm{e}-4(0.6 \mathrm{e}-6)$ & $0.24 \mathrm{e}-4(0.2 \mathrm{e}-6)$ & $0.25 \mathrm{e}-4(0.2 \mathrm{e}-6)$ & $0.40 \mathrm{e}-4(0.4 \mathrm{e}-6)$ & $0.34 \mathrm{e}-4(0.3 \mathrm{e}-6)$ \\
$1-0 S(2)$ & 2.0338 & $0.85 \mathrm{e}-5(0.6 \mathrm{e}-6)$ & $0.68 \mathrm{e}-5(0.2 \mathrm{e}-6)$ & $0.72 \mathrm{e}-5(0.3 \mathrm{e}-6)$ & $0.11 \mathrm{e}-4(0.4 \mathrm{e}-6)$ & $0.97 \mathrm{e}-5(0.3 \mathrm{e}-6)$ \\
$1-0 S(6)$ & 1.7880 & $0.13 \mathrm{e}-5(0.5 \mathrm{e}-6)$ & $0.14 \mathrm{e}-5(0.1 \mathrm{e}-6)$ & $0.14 \mathrm{e}-5(0.7 \mathrm{e}-7)$ & $0.19 \mathrm{e}-5(0.2 \mathrm{e}-6)$ & $0.17 \mathrm{e}-5(0.2 \mathrm{e}-6)$ \\
$1-0 S(7)$ & 1.7480 & $0.27 \mathrm{e}-5(0.4 \mathrm{e}-6)$ & $0.17 \mathrm{e}-5(0.1 \mathrm{e}-6)$ & $0.18 \mathrm{e}-5(0.7 \mathrm{e}-7)$ & $0.21 \mathrm{e}-5(0.2 \mathrm{e}-6)$ & $0.16 \mathrm{e}-5(0.2 \mathrm{e}-6)$ \\
$1-0 S(8)$ & 1.7147 & $<0.7 \mathrm{e}-6$ & $0.34 \mathrm{e}-6(0.1 \mathrm{e}-6)$ & $0.27 \mathrm{e}-6(0.8 \mathrm{e}-7)$ & - & $<0.4 \mathrm{e}-6$ \\
$1-0 S(9)$ & 1.6877 & $<0.7 \mathrm{e}-6$ & $0.36 \mathrm{e}-6(0.1 \mathrm{e}-6)$ & $0.27 \mathrm{e}-6(0.6 \mathrm{e}-7)$ & $0.54 \mathrm{e}-6(0.2 \mathrm{e}-6)$ & $0.46 \mathrm{e}-6(0.2 \mathrm{e}-6)$ \\
$2-1 S(1)$ & 2.2477 & $0.44 \mathrm{e}-5(0.6 \mathrm{e}-6)$ & $0.33 \mathrm{e}-5(0.2 \mathrm{e}-6)$ & $0.37 \mathrm{e}-5(0.3 \mathrm{e}-6)$ & $0.50 \mathrm{e}-5(0.4 \mathrm{e}-6)$ & $0.41 \mathrm{e}-5(0.4 \mathrm{e}-6)$ \\
$2-1 S(2)$ & 2.1542 & $0.19 \mathrm{e}-5(0.6 \mathrm{e}-6)$ & $0.80 \mathrm{e}-6(0.2 \mathrm{e}-6)$ & $0.13 \mathrm{e}-5(0.2 \mathrm{e}-6)$ & $0.17 \mathrm{e}-5(0.3 \mathrm{e}-6)$ & $<0.15 \mathrm{e}-5$ \\
$2-1 S(3)$ & 2.0735 & $0.33 \mathrm{e}-5(0.6 \mathrm{e}-6)$ & $0.27 \mathrm{e}-5(0.2 \mathrm{e}-6)$ & $0.27 \mathrm{e}-5(0.2 \mathrm{e}-6)$ & $0.41 \mathrm{e}-5(0.4 \mathrm{e}-6)$ & $0.23 \mathrm{e}-5(0.3 \mathrm{e}-6)$ \\
$3-2 S(3)$ & 2.2014 & $0.29 \mathrm{e}-5(0.9 \mathrm{e}-6)$ & $0.64 \mathrm{e}-6(0.2 \mathrm{e}-6)$ & $0.72 \mathrm{e}-6(0.3 \mathrm{e}-6)$ & - & $0.13 \mathrm{e}-5(0.4 \mathrm{e}-6)$ \\
$1-0 Q(1)$ & 2.4066 & $0.40 \mathrm{e}-4(0.4 \mathrm{e}-5)$ & $0.27 \mathrm{e}-4(0.1 \mathrm{e}-5)$ & $0.29 \mathrm{e}-4(0.1 \mathrm{e}-5)$ & $0.18 \mathrm{e}-4(0.3 \mathrm{e}-5)$ & $0.18 \mathrm{e}-4(0.3 \mathrm{e}-5)$ \\
$1-0 Q(2)$ & 2.4134 & $0.13 \mathrm{e}-4(0.4 \mathrm{e}-5)$ & $0.16 \mathrm{e}-4(0.2 \mathrm{e}-5)$ & $0.13 \mathrm{e}-4(0.1 \mathrm{e}-5)$ & $0.19 \mathrm{e}-4(0.3 \mathrm{e}-5)$ & $0.19 \mathrm{e}-4(0.3 \mathrm{e}-5)$ \\
$1-0 Q(3)$ & 2.4237 & $0.22 \mathrm{e}-4(0.4 \mathrm{e}-5)$ & $0.24 \mathrm{e}-4(0.1 \mathrm{e}-5)$ & $0.26 \mathrm{e}-4(0.1 \mathrm{e}-5)$ & $0.20 \mathrm{e}-4(0.3 \mathrm{e}-5)$ & $0.20 \mathrm{e}-4(0.3 \mathrm{e}-5)$ \\
$1-0 Q(4)$ & 2.4375 & $0.21 \mathrm{e}-4(0.5 \mathrm{e}-5)$ & $0.19 \mathrm{e}-4(0.1 \mathrm{e}-5)$ & $0.13 \mathrm{e}-4(0.1 \mathrm{e}-5)$ & $0.21 \mathrm{e}-4(0.4 \mathrm{e}-5)$ & $0.20 \mathrm{e}-4(0.3 \mathrm{e}-5)$ \\
$1-0 Q(5)$ & 2.4548 & $0.36 \mathrm{e}-4(0.4 \mathrm{e}-5)$ & $0.32 \mathrm{e}-4(0.1 \mathrm{e}-5)$ & $0.33 \mathrm{e}-4(0.1 \mathrm{e}-5)$ & $0.52 \mathrm{e}-4(0.3 \mathrm{e}-5)$ & $0.47 \mathrm{e}-4(0.3 \mathrm{e}-5)$ \\
\hline
\end{tabular}

Line intensities given in $\mathrm{Jy} \mu \mathrm{m}$ (to convert to $\mathrm{W} \mathrm{m}^{-2}$ multiply by $3 \times 10^{-24} / \lambda^{2}$ ). Errors are given in brackets. Vacuum wavelengths in column two are in microns. 